\title{
Reformulating Unsupervised Style Transfer as Paraphrase Generation
}

\author{
Kalpesh Krishna ${ }^{\oplus}$ \\ John Wieting $\diamond$ \\ Mohit Iyyer ${ }^{\infty}$ \\ ^University of Massachusetts Amherst, ${ }^{\diamond}$ Carnegie Mellon University \\ \{kalpesh, miyyer\}ecs.umass.edu \\ jwieting@cs.cmu.edu
}

Project Page: http://style.cs.umass.edu

\begin{abstract}
Modern NLP defines the task of style transfer as modifying the style of a given sentence without appreciably changing its semantics, which implies that the outputs of style transfer systems should be paraphrases of their inputs. However, many existing systems purportedly designed for style transfer inherently warp the input's meaning through attribute transfer, which changes semantic properties such as sentiment. In this paper, we reformulate unsupervised style transfer as a paraphrase generation problem, and present a simple methodology based on fine-tuning pretrained language models on automatically generated paraphrase data. Despite its simplicity, our method significantly outperforms state-of-the-art style transfer systems on both human and automatic evaluations. We also survey 23 style transfer papers and discover that existing automatic metrics can be easily gamed and propose fixed variants. Finally, we pivot to a more real-world style transfer setting by collecting a large dataset of $15 \mathrm{M}$ sentences in 11 diverse styles, which we use for an in-depth analysis of our system.
\end{abstract}

\section{Introduction}

The task of style transfer on text data involves changing the style of a given sentence while preserving its semantics. ${ }^{1}$ Recent work in this area conflates style transfer with the related task of attribute transfer (Subramanian et al., 2019; He et al., 2020), in which modifications to attributespecific content words (e.g., those that carry sentiment) warp both stylistic and semantic properties of a sentence (Preotiuc-Pietro et al., 2016). Attribute transfer has been criticized for its limited real-world applications: Pang (2019) argue that se-

\footnotetext{
${ }^{1}$ We use the quasi-paraphrase definition of semantic equivalence from Bhagat and Hovy (2013) throughout this paper. We loosely define style as patterns in lexical and syntactic choice within the space of quasi-paraphrases.
}

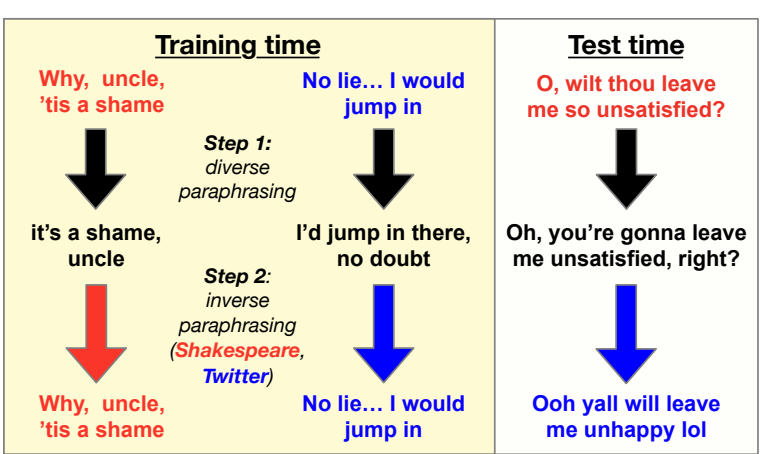

Figure 1: During training, STRAP applies a diverse paraphraser to an input sentence and passes the result through a style-specific inverse paraphraser to reconstruct the input. At test time, we perform style transfer by swapping out different inverse paraphrase models (Shakespeare $\rightarrow$ Twitter shown here). All generated sentences shown here are actual outputs from STRAP.

mantic preservation is critical for author obfuscation (Shetty et al., 2018), data augmentation (Xie et al., 2019; Kaushik et al., 2020), text simplification (Xu et al., 2015), writing assistance (Heidorn, 2000). Moreover, semantic preservation (via paraphrases) has several applications like better translation evaluation (Sellam et al., 2020; Freitag et al., 2020) and adversarial defenses (Iyyer et al., 2018).

We propose to improve semantic preservation in style transfer by modeling the task as a controlled paraphrase generation problem. Our unsupervised method (Style Transfer via Paraphrasing, or STRAP) requires no parallel data between different styles and proceeds in three simple stages:

1. Create pseudo-parallel data by feeding sentences from different styles through a diverse paraphrase model (Figure 1, left).

2. Train style-specific inverse paraphrase models that convert these paraphrased sentences back into the original stylized sentences.

3. Use the inverse paraphraser for a desired style to perform style transfer (Figure 1, right). 
Our approach requires none of the finicky ${ }^{2}$ modeling paradigms popular in style transfer research no reinforcement learning (Luo et al., 2019), variational inference (He et al., 2020), or autoregressive sampling during training (Subramanian et al., 2019). Instead, we implement the first two stages of our pipeline by simply fine-tuning a pretrained GPT-2 language model (Radford et al., 2019).

Despite its simplicity, STRAP significantly outperforms the state of the art on formality transfer and Shakespeare author imitation datasets by $\mathbf{2 - 3 x}$ on automatic evaluations and 4-5x on human evaluations. We further show that only 3 out of 23 prior style transfer papers properly evaluate their models: in fact, a naïve baseline that randomly chooses to either copy its input or retrieve a random sentence written in the target style outperforms prior work on poorly-designed metrics.

Finally, we take a step towards real-world style transfer by collecting a large dataset CDS (Corpus of Diverse Styles) of 15M English sentences spanning 11 diverse styles, including the works of James Joyce, romantic poetry, tweets, and conversational speech. CDS is orders of magnitude larger and more complex than prior benchmarks, which generally focus on transferring between just two styles. We analyze STRAP's abilities on CDS, and will release it as a benchmark for future research. In summary, our contributions are:

(1) a simple approach to perform lexically and syntactically diverse paraphrasing with pretrained language models;

(2) a simple unsupervised style transfer method that models semantic preservation with our paraphraser and significantly outperforms prior work;

(3) a critique of existing style transfer evaluation based on a naïve baseline that performs on par with prior work on poorly designed metrics;

(4) a new benchmark dataset that contains $15 \mathrm{M}$ sentences from 11 diverse styles.

\section{Style Transfer via Paraphrasing}

We loosely define style as common patterns of lexical choice and syntactic constructions that are distinct from the content of a sentence, following prior work (Hovy, 1987; DiMarco and Hirst, 1993; Green and DiMarco, 1993; Kabbara and Cheung, 2016). While we acknowledge this distinction is

\footnotetext{
${ }^{2}$ For example, reproducing deep RL methods is challenging (Henderson et al., 2018), vanilla adversarial training is unstable (Arjovsky et al., 2017), and VAEs suffer from posterior collapse (Bowman et al., 2016).
}

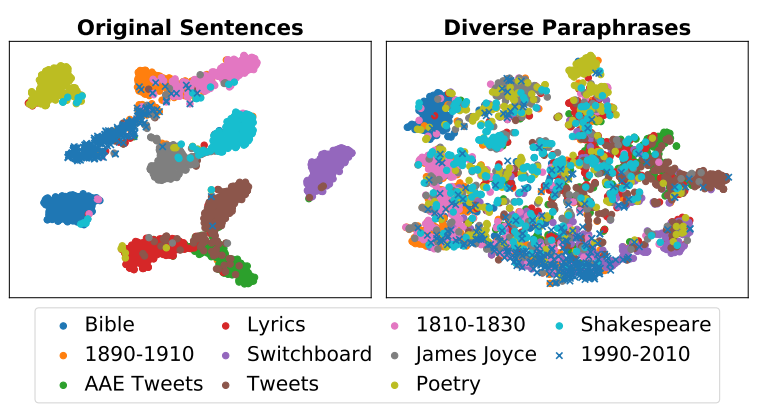

Figure 2: Diverse paraphrasing normalizes sentences by removing stylistic identifiers. We cluster validation sentences from our CDS dataset by applying $t$-SNE to [CLS] vectors from a RoBERTa style classifier. The original sentences (left) form distinct clusters, while the paraphrased sentences (right) do not, showing the stylized text has been normalized.

not universally accepted, ${ }^{3}$ this treatment is critical to unlock several real-world applications of style transfer (as argued in Section 1). Unfortunately, many modern style transfer systems do not respect this definition: a human evaluation (Table 2) shows that fewer than $\mathbf{2 5 \%}$ of style-transferred sentences from two state-of-the-art systems (Subramanian et al., 2019; He et al., 2020) on formality transfer were rated as paraphrases of their inputs.

Motivated by this result, we reformulate style transfer as a controlled paraphrase generation task. We call our method STRAP, or Style Transfer via Paraphrasing. STRAP operates within an unsupervised setting: we have raw text from distinct target styles, but no access to parallel sentences paraphrased into different styles. To get around this lack of data, we create pseudo-parallel sentence pairs using a paraphrase model (Section 2.1) trained to maximize output diversity (Section 2.4). Intuitively, this paraphrasing step normalizes the input sentence by stripping away information that is predictive of its original style (Figure 2). The normalization effect allows us to train an inverse paraphrase model specific to the original style, which attempts to generate the original sentence given its normalized version (Section 2.2). Through this process, the model learns to identify and produce salient features of the original style without unduly warping the input semantics.

\subsection{Creating pseudo-parallel training data}

The first stage of our approach involves normalizing input sentences by feeding them through a

\footnotetext{
${ }^{3}$ For example, Eckert (2008) considers style and semantics to be inseparable; while Meyerhoff (2015) considers style to be intra-speaker variation in different social contexts
} 
diverse paraphrase model. Consider a corpus of sentences from multiple styles, where the set of all sentences from style $i$ is denoted by $\mathbf{X}^{i}$. We first generate a paraphrase $\mathbf{z}$ for every sentence $\mathbf{x} \in \mathbf{X}^{i}$ using a pretrained paraphrase model $f_{\text {para }}$,

$$
\mathbf{z}=f_{\text {para }}(\mathbf{x}) \text { where } \mathbf{x} \in \mathbf{X}^{i} .
$$

This process results in a dataset $\mathbf{Z}^{i}$ of normalized sentences and allows us to form a pseudo-parallel corpus $\left(\mathbf{X}^{i}, \mathbf{Z}^{i}\right)$ between each original sentence and its paraphrased version. Figure 2 shows that this paraphrasing process has a powerful style normalization effect for our instantiation of $f_{\text {para }}$.

\subsection{Style transfer via inverse paraphrasing}

We use this pseudo-parallel corpus to train a stylespecific model that attempts to reconstruct the original sentence $\mathbf{x}$ given its paraphrase $\mathbf{z}$. Since $f_{\text {para }}$ removes style identifiers from its input, the intuition behind this inverse paraphrase model is that it learns to insert stylistic features through the reconstruction process. Formally, the inverse paraphrase model $f_{\text {inv }}^{i}$ for style $i$ learns to reconstruct ${ }^{4}$ the original corpus $\mathbf{X}^{i}$ using the standard language modeling objective with cross-entropy loss $\mathcal{L}_{\mathrm{CE}}$,

$$
\begin{aligned}
\overline{\mathbf{x}} & =f_{\text {inv }}^{i}(\mathbf{z}) \text { where } \mathbf{z} \in \mathbf{Z}^{i} \\
\text { loss } & =\sum_{\mathbf{x} \in \mathbf{X}^{i}} \mathcal{L}_{\mathrm{CE}}(\mathbf{x}, \overline{\mathbf{x}})
\end{aligned}
$$

During inference, given an arbitrary sentence $\mathbf{s}$ (in any particular style), we convert it to a sentence $\overline{\mathbf{s}}^{j}$ in target style $j$ using a two-step process of style normalization with $f_{\text {para }}$ followed by stylization with the inverse paraphraser $f_{\text {inv }}^{j}$, as in

$$
\overline{\mathbf{s}}^{j}=f_{\text {inv }}^{j}\left(f_{\text {para }}(\mathbf{s})\right) .
$$

\subsection{Paraphraser implementation with GPT-2}

We fine-tune the large-scale pretrained GPT2-large language model (Radford et al., 2019) to implement both the paraphraser $f_{\text {para }}$ and inverse paraphrasers $f_{\text {inv }}^{i}$ for each style. ${ }^{5}$ Starting from a pretrained LM improves both output fluency and generalization to small style-specific datasets (Section 5). We use the encoder-free seq2seq modeling approach

\footnotetext{
${ }^{4}$ This process resembles denoising autoencoders (Vincent et al., 2008; Lample et al., 2018, DAE): $f_{\text {para }}$ acts as a semantic preserving noise function; $f_{\text {inv }}^{i}$ reconstructs the input.

${ }^{5}$ We fine-tune a separate GPT- 2 model $f_{\text {inv }}^{i}$ per style. Section 5 shows that this outperforms a single inverse paraphraser shared across all styles with style input.
}

described in Wolf et al. (2018), where input and output sequences are concatenated together with a separator token. We use Hugging Face's Transformers library (Wolf et al., 2019) to implement our models; see Appendix A.2 for more details about the architecture \& hyperparameters.

\subsection{Promoting diversity by filtering data}

The final piece to our approach is how we choose training data for the paraphrase model $f_{\text {para }}$. We discover that maximizing lexical and syntactic diversity of the output paraphrases is crucial for effective style normalization (Section 5, 6). We promote output diversity by training $f_{\text {para }}$ on an aggressivelyfiltered subset of PARANMT-50M (Wieting and Gimpel, 2018), a large corpus of backtranslated text. Specifically, we apply three filters: (1) removing sentence pairs with more than $50 \%$ trigram or unigram overlap to maximize lexical diversity and discourage copying; (2) removing pairs with lower than $50 \%$ reordering of shared words, measured by Kendall's tau (Kendall, 1938), to promote syntactic diversity; and (3) removing pairs with low semantic similarity, measured by the SIM model from Wieting et al. (2019). ${ }^{6}$ After applying these filters, our training data size shrinks from $50 \mathrm{M}$ to $75 \mathrm{~K}$ sentence pairs, which are used to fine-tune GPT-2; see Appendix A.1 for more details about the filtering process and its effect on corpus size.

\section{Evaluating style transfer}

Providing a meaningful comparison of our approach to existing style transfer systems is difficult because of (1) poorly-defined automatic and human methods for measuring style transfer quality (Pang, 2019; Mir et al., 2019; Tikhonov et al., 2019), and (2) misleading (or absent) methods of aggregating three individual metrics (transfer accuracy, semantic similarity and fluency) into a single number. In this section, we describe the flaws in existing metrics and their aggregation (the latter illustrated through a naïve baseline), and we propose a new evaluation methodology to fix these issues.

\subsection{Current state of style transfer evaluation}

We conduct a survey of $\mathbf{2 3}$ previously-published style transfer papers (more details in Appendix A.9), which reveals three common

\footnotetext{
${ }^{6}$ This model achieves strong performance on semantic textual similarity (STS) SemEval benchmarks (Agirre et al., 2016). We remove all pairs with a score lower than 0.7 .
} 
properties on which style transfer systems are evaluated. Here, we discuss how prior work implements evaluations for each of these properties and propose improved implementations to address some of their downsides.

Transfer accuracy (ACC): Given an output sentence $\overline{\mathbf{s}}^{j}$ and a target style $j$, a common way of measuring transfer success is to train a classifier to identify the style of a transferred sentence and report its accuracy ACC on generated sentences (i.e., whether $\overline{\mathbf{s}}^{j}$ has a predicted style of $j$ ). 14 of 23 surveyed papers implement this style classifier with a 1-layer CNN (Kim, 2014). However, recent large Transformers like BERT (Devlin et al., 2019) significantly outperform CNNs on most NLP tasks, including style classification. Thus, we build our style classifier by fine-tuning RoBERTa-large (Liu et al., 2019) on all our datasets, leading to significantly more reliable ACC evaluation. ${ }^{7}$

Semantic similarity (SIM): A style transfer system can achieve high ACC scores without maintaining the semantics of the input sentence, which motivates also measuring how much a transferred sentence deviates in meaning from the input. 15 / 23 surveyed papers use $n$-gram metrics like BLEU (Papineni et al., 2002) against reference sentences, often along with self-BLEU with the input, to evaluate semantic similarity. Using BLEU in this way has many problems, including (1) unreliable correlations between $n$-gram overlap and human evaluations of semantic similarity (Callison-Burch et al., 2006), (2) discouraging output diversity (Wieting et al., 2019), and (3) not upweighting important semantic words over other words (Wieting et al., 2019; Wang et al., 2020). These issues motivate us to measure semantic similarity using the subword embedding-based SIM model of Wieting et al. (2019), which performs well on semantic textual similarity (STS) benchmarks in SemEval workshops (Agirre et al., 2016). ${ }^{8}$

Fluency (FL): A system that produces ungrammatical outputs can still achieve high scores on both ACC and SIM, motivating a separate measure for fluency. Only 10 out of 23 surveyed papers did a fluency evaluation; 9 of which used language model perplexity, which is a poor measure because

\footnotetext{
${ }^{7}$ The RoBERTa style classifier, built with fairseq (Ott et al., 2019), achieves a test accuracy of $90.4 \%$ on the Shakespeare data(vs $83.5 \%$ for CNN) and $94.8 \%$ on the Formality data (vs 92.4\%). The datasets are introduced in Section 4.1.

${ }^{8}$ For reference, we evaluate with BLEU in Appendix A.5.
}

(1) it is unbounded and (2) unnatural sentences with common words tend to have low perplexity (Mir et al., 2019; Pang, 2019). To tackle this we replace perplexity with the accuracy of a RoBERTa-large classifier trained on the CoLA corpus (Warstadt et al., 2019), which contains sentences paired with grammatical acceptability judgments. In Table 1, we show that our classifier marks most reference sentences as fluent, confirming its validity. ${ }^{9}$

Human evaluation: As automatic evaluations are insufficient for evaluating text generation (Liu et al., 2016; Novikova et al., 2017), 17 out of 23 surveyed style transfer papers also conduct human evaluation. In our work, we evaluate SIM and FL using human evaluations. ${ }^{10}$ As we treat style transfer as a paraphrase generation task, we borrow the three-point scale used previously to evaluate paraphrases (Kok and Brockett, 2010; Iyyer et al., 2018), which jointly captures SIM and FL. Given the original sentence and the transferred sentence, annotators on Amazon Mechanical Turk can choose one of three options: $\mathbf{0}$ for no paraphrase relationship; 1 for an ungrammatical paraphrase; and $\mathbf{2}$ for a grammatical paraphrase. A total of 150 sentence pairs were annotated per model, with three annotators per pair. More details on our setup, payment \& agreement are provided in Appendix A.10.

\subsection{Aggregation of Metrics}

So far, we have focused on individual implementations of ACC, SIM, and FL. After computing these metrics, it is useful to aggregate them into a single number to compare the overall style transfer quality across systems (Pang, 2019). However, only 5 out of the 23 papers aggregate these metrics, either at the corpus level (Xu et al., 2018; Pang and Gimpel, 2019) or sentence level ( $\mathrm{Li}$ et al., 2018). Even worse, the corpus-level aggregation scheme can be easily gamed. Here, we describe a naïve system that outperforms state-of-the-art style transfer systems when evaluated using corpus-level aggregation, and we present a new sentence-level aggregation metric that fixes the issue.

The issue with corpus-level aggregation: Aggregating ACC, SIM, and FL is inherently difficult

\footnotetext{
${ }^{9}$ Mir et al. (2019) also recommended a similar method to evaluate fluency instead of perplexity, where they train classifiers to distinguish between machine / human sentences.

${ }^{10} \mathrm{We}$ do not conduct human evaluations for ACC since style classification is difficult for an untrained crowdsourced worker unfamiliar with the set of target styles.
} 
because they are inversely correlated with each other (Pang, 2019). Prior work has combined these three scores into a single number using geometric averaging (Xu et al., 2018) or learned weights (Pang and Gimpel, 2019). However, the aggregation is computed after averaging each metric independently across the test set (corpus-level aggregation), which is problematic since systems might generate sentences that optimize only a subset of metrics. For example, a Shakespeare style transfer system could output Wherefore art thou Romeo? regardless of its input and score high on ACC and FL, while a model that always copies its input would score well on SIM and FL (Pang, 2019).

A Naïve Style Transfer System: To concretely illustrate the problem, we design a naïve baseline that exactly copies its input with probability $p$ and chooses a random sentence from the target style corpus for the remaining inputs, where $p$ is tuned on the validation set. ${ }^{11}$ When evaluated using geometric mean corpus-level aggregation (GM column of Table 1) this system outperforms state of the art methods (UNMT, DSLM) on the Formality dataset despite not doing any style transfer at all!

Proposed Metric: A good style transfer system should jointly optimize all metrics. The strong performance of the naïve baseline with corpus-level aggregation indicates that metrics should be combined at the sentence level before averaging them across the test set (sentence aggregation). Unfortunately, only 3 out of 23 surveyed papers measure absolute performance after sentence-level aggregation, and all of them use the setup of Li et al. (2018), which is specific to human evaluation with Likert scales. We propose a more general alternative,

$$
J(\operatorname{ACC}, \operatorname{SIM}, \mathrm{FL})=\sum_{x \in \mathbf{X}} \frac{\operatorname{ACC}(x) \cdot \operatorname{SIM}(x) \cdot \operatorname{FL}(x)}{|\mathbf{X}|}
$$

where $x$ is a sentence from a test corpus $\mathbf{X}$. We treat ACC and FL at a sentence level as a binary judgement, ensuring incorrectly classified or disfluent sentences are automatically assigned a score of 0 . As a sanity check, our naïve system performs extremely poorly on this new metric (Table 1), as input copying will almost always yield an ACC of zero, while random retrieval results in low SIM.

\footnotetext{
${ }^{11} p=0.4 / 0.5$ for Formality / Shakespeare datasets.
}

\section{Experiments \& Results}

We evaluate our method (STRAP) on two existing style transfer datasets, using the evaluation methodology proposed in Section 3. Our system significantly outperforms state of the art methods and the naïve baseline discussed in Section 3.2.

\subsection{Datasets}

We focus exclusively on semantics-preserving style transfer tasks, which means that we do not evaluate on attribute transfer datasets such as sentiment, gender, and political transfer. Specifically, we use two standard benchmark datasets for Shakespeare author imitation and formality transfer to compare STRAP against prior work. While both datasets contain parallel data, we only use it to automatically evaluate our model outputs; for training, we follow prior work by using the non-parallel trainvalidation-test splits from He et al. (2020).

The Shakespeare author imitation dataset $(\mathrm{Xu}$ et al., 2012) contains 37k training sentences from two styles - William Shakespeare's original plays, and their modernized versions. Shakespeare's plays are written in Early Modern English, which has a significantly different lexical (e.g., thou instead of you) and syntactic distribution compared to modern English. Our second dataset is Formality transfer (Rao and Tetreault, 2018), which contains 105k sentences, also from two styles. Sentences are written either in formal or informal modern English. Unlike formal sentences, informal sentences tend to have more misspellings, short forms ( $u$ instead of you), and non-standard usage of punctuation.

\subsection{Comparisons against prior work}

We compare STRAP on the Shakespeare / Formality datasets against the following baselines:

- COPY: a lower bound that simply copies its input, which has been previously used in prior work (Subramanian et al., 2019; Pang, 2019)

- NAÏVE: our method from Section 3.2 that randomly either copies its input or retrieves a sentence from the target style

- REF: an upper bound computed by evaluating reference sentences using our metrics

- UNMT: unsupervised neural machine translation from Subramanian et al. (2019)

- DLSM: the deep latent sequence model from 


\begin{tabular}{lrrrrr|rrrrr}
\hline Model & \multicolumn{4}{c|}{ Formality (GYAFC) } & \multicolumn{5}{c}{ Shakespeare } \\
& ACC & SIM & FL & GM(A,S,F) & $J(\mathrm{~A}, \mathrm{~S}, \mathrm{~F})$ & ACC & SIM & FL & GM(A,S,F) & $J(\mathrm{~A}, \mathrm{~S}, \mathrm{~F})$ \\
\hline COPY & 5.2 & 80.1 & 88.4 & 33.3 & 4.2 & 9.6 & 67.1 & 79.1 & 37.1 & 7.2 \\
NAÏVE & 58.9 & 38.9 & 89.1 & 58.9 & 7.3 & 49.9 & 34.9 & 78.9 & 51.6 & 4.1 \\
REF & 93.3 & 100 & 89.7 & 94.2 & 83.8 & 90.4 & 100 & 79.1 & 89.4 & 70.5 \\
\hline UNMT (2019) & $\mathbf{7 8 . 5}$ & 49.1 & 52.5 & 58.7 & 20.0 & 70.5 & 37.5 & 49.6 & 50.8 & 14.6 \\
DLSM (2020) & 78.0 & 47.7 & 53.7 & 58.5 & 18.6 & 71.1 & 43.5 & 49.4 & 53.5 & 16.3 \\
\hline STRAP $(p=0.0)$ & 67.7 & $\mathbf{7 2 . 5}$ & $\mathbf{9 0 . 4}$ & $\mathbf{7 6 . 3}$ & $\mathbf{4 5 . 5}$ & 71.7 & $\mathbf{5 6 . 4}$ & $\mathbf{8 5 . 2}$ & $\mathbf{7 0 . 1}$ & $\mathbf{3 4 . 7}$ \\
STRAP $(p=0.6)$ & 70.7 & 69.9 & 88.5 & 75.9 & 44.5 & 75.7 & 53.7 & 82.7 & 69.5 & 33.5 \\
STRAP $(p=0.9)$ & 76.8 & 62.9 & 77.4 & 72.0 & 38.3 & $\mathbf{7 9 . 8}$ & 47.6 & 71.7 & 64.8 & 27.5 \\
\hline
\end{tabular}

Table 1: Automatic evaluation of our method STRAP (using different $p$ values for nucleus sampling) against prior state-of-the-art methods (UNMT, DLSM), lower bound baselines (COPY, NAÏVE) and reference sentences (REF). STRAP significantly outperforms prior work, especially on our proposed $J(\cdot)$ metric. GM is the geometric mean.

\begin{tabular}{llrrrr}
\hline Dataset & Model & ACC & SIM & $J(\mathrm{~A}, \mathrm{~S})$ & $J(\mathrm{~A}, \mathrm{~S}, \mathrm{~F})$ \\
\hline Form. & UNMT & 77.3 & 22.7 & 14.7 & 7.3 \\
& DLSM & 78.0 & 24.0 & 15.3 & 10.0 \\
\cline { 2 - 5 } & $p=0.0$ & 71.3 & $\mathbf{7 6 . 0}$ & $\mathbf{5 4 . 7}$ & $\mathbf{4 1 . 3}$ \\
& $p=0.9$ & $\mathbf{7 9 . 3}$ & 56.7 & 46.0 & 28.0 \\
\hline Shak. & UNMT & 69.3 & 20.7 & 10.0 & 7.3 \\
& DLSM & 65.3 & 37.3 & 21.3 & 9.3 \\
\cline { 2 - 5 } & $p=0.0$ & 70.7 & $\mathbf{7 9 . 3}$ & $\mathbf{5 6 . 0}$ & $\mathbf{4 7 . 3}$ \\
& $p=0.9$ & $\mathbf{7 4 . 7}$ & 54.0 & 38.0 & 24.7 \\
\hline
\end{tabular}

Table 2: Human evaluation of STRAP with greedy decoding $(p=0.0)$ and nucleus sampling $(p=0.9)$ shows large improvements (4-5x) on both the Formality (Form.) and Shakespeare (Shak.) datasets. Details on metric calculations are provided in Appendix A.10.

He et al. (2020), which is currently state-ofthe-art on both datasets. ${ }^{12}$

STRAP significantly outperforms the prior state of the art (DLSM) on automatic metrics (Table 1) with a $J(\cdot)$ score of 45.5 (vs 18.6) on Formality and 34.7 (vs 16.3) on Shakespeare. The improvements are even larger when SIM and FL are measured through human evaluations (Table 2): in this setting, STRAP achieves 41.3 (vs 10.0) on Formality and 47.3 (vs 9.3) on Shakespeare. Across the board, STRAP significantly improves in SIM and FL while maintaining similar ACC. Finally, the large gap between REF and STRAP on automatic metrics provides exciting avenues for future research. ${ }^{13}$

\footnotetext{
${ }^{12}$ We use the implementations of both UNMT and DLSM made publicly available by $\mathrm{He}$ et al. (2020), and we verify that their UNMT model performs on par with reported sentiment transfer numbers in Subramanian et al. (2019). The original code of Subramanian et al. (2019) has not been open-sourced.

${ }^{13}$ Results with other metrics such as BLEU, as well as comparisons against several other baselines like Li et al. (2018); Prabhumoye et al. (2018); Luo et al. (2019); Dai et al. (2019); Sudhakar et al. (2019) are provided in Appendix A.5. STRAP significantly outperforms all prior work.
}

\begin{tabular}{llrrrr}
\hline Dataset & Model & ACC & SIM & FL & $J(\mathrm{~A}, \mathrm{~S}, \mathrm{~F})$ \\
\hline Form. & STRAP & 67.7 & 72.5 & 90.4 & 45.5 \\
& - Inf. PP & 27.5 & 78.5 & 88.2 & 20.7 \\
& - Mult. PP & 63.1 & 72.0 & 90.8 & 42.3 \\
& - Div. PP & 61.2 & 79.5 & 88.7 & 43.8 \\
& - GPT2 & 84.6 & 43.8 & 61.7 & 23.1 \\
& GPT2-md & 71.0 & 70.7 & 88.6 & 45.8 \\
& GPT2-sm & 69.1 & 68.6 & 87.6 & 42.9 \\
\hline Shak. & STRAP & 71.7 & 56.4 & 85.2 & 34.7 \\
& - Inf. PP & 40.1 & 66.1 & 76.3 & 23.3 \\
& - Mult. PP & 45.9 & 56.5 & 91.1 & 24.8 \\
& - Div. PP & 49.7 & 64.4 & 82.9 & 28.2 \\
& - GPT2 & 75.6 & 26.7 & 66.9 & 13.6 \\
& GPT2-md & 73.4 & 54.0 & 86.4 & 34.3 \\
& GPT2-sm & 68.0 & 53.2 & 84.6 & 31.5 \\
\hline
\end{tabular}

Table 3: Ablation study using automatic metrics on the Formality (Form.) and Shakespeare (Shak.) datasets.

\section{Ablation studies}

In this section, we perform several ablations on STRAP to understand which of its components contribute most to its improvements over baselines. Overall, these ablations validate the importance of both paraphrasing and pretraining for style transfer.

Paraphrase diversity improves ACC: How critical is diversity in the paraphrase generation step? While our implementation of $f_{\text {para }}$ is trained on data that is heavily-filtered to promote diversity, we also build a non-diverse paraphrase model by removing this diversity filtering of PARANMT$50 \mathrm{M}$ but keeping all other experimental settings identical. In Table 3, the - Div. PP rows show a drop in ACC across both datasets as well as higher SIM, which in both cases results in a lower $J(\cdot)$ score. A qualitative inspection reveals that the decreased ACC and increased SIM are both due to a greater degree of input copying, which motivates the importance of diversity.

Paraphrasing during inference improves ACC: 
The diverse paraphraser $f_{\text {para }}$ is obviously crucial to train our model, as it creates pseudo-parallel data for training $f_{\mathrm{inv}}^{i}$, but is it necessary during inference? We try directly feeding in the original sentence (without the initial paraphrasing step) to the inverse paraphrase model $f_{\text {inv }}^{i}$ during inference, shown in the $-I n f$. PP row of Table 3. While SIM and FL are largely unaffected, there is a large drop in ACC, bringing down the overall score (45.5 to 20.7 in Formality, 34.7 to 23.3 in Shakespeare). This supports our hypothesis that the paraphrasing step is useful for normalizing the input.

LM pretraining is crucial for SIM and FL: As we mainly observe improvements on FL and SIM compared to prior work, a natural question is how well does STRAP perform without large-scale LM pretraining? We run an ablation study by replacing the GPT-2 implementations of $f_{\text {para }}$ and $f_{\text {inv }}^{i}$ with LSTM seq2seq models, which are trained with global attention (Luong et al., 2015) using OpenNMT (Klein et al., 2017) with mostly default hyperparameters. ${ }^{14}$ As seen in the $-G P T 2$ row of Table 3, this model performs competitively with the UNMT / DLSM models on J(ACC,SIM,FL), which obtain 20.0 / 18.6 on Formality (Table 1), respectively. However, it is significantly worse than STRAP, with large drops in SIM and FL. ${ }^{15}$ This result shows the merit of both our algorithm and the boost that LM pretraining provides. ${ }^{16}$

Nucleus sampling trades off ACC for SIM: While our best performing system uses a greedy decoding strategy, we experiment with nucleus sampling (Holtzman et al., 2020) by varying the nucleus $p$ value in both Table 1 and Table 2. As expected, higher $p$ improves diversity and trades off increased ACC for lowered SIM. We find that $p=0.6$ is similar to greedy decoding on $J(\cdot)$ metrics, but higher $p$ values degrade performance.

Multiple inverse paraphrasers perform better than a single style-conditional model: Finally, we explore a more parameter-efficient alternative to training a separate inverse paraphrase model per style. Prior work in conditioned language models

\footnotetext{
${ }^{14}$ The only hyperparameter we tune is the learning rate schedule. More details in Appendix A.4.

${ }^{15}$ A qualitative inspection of outputs confirms the LSTM struggles to maintain semantics. We suspect this is due to lack of training data $(<75 \mathrm{~K}$ pairs) to learn a powerful paraphraser.

${ }^{16}$ Additionally, we note that weaker pretrained language models like GPT2-medium $(G P T 2-m d)$ perform similarly to GPT2-large, while GPT2-small (GPT2-sm) is notably worse.
}

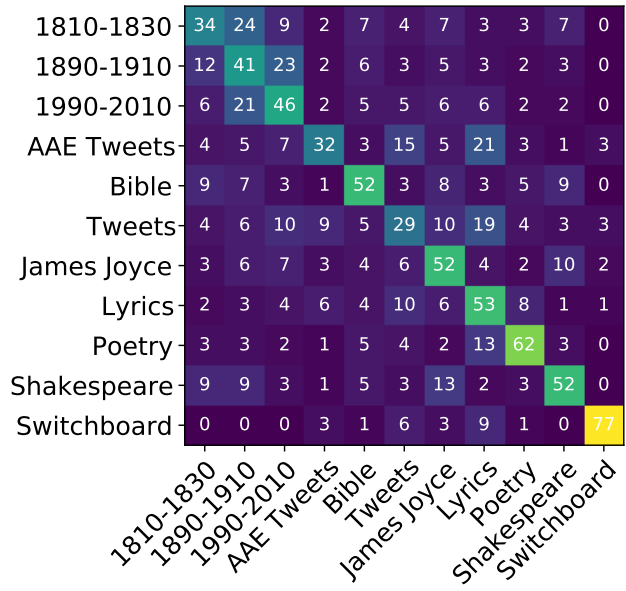

Figure 3: Classifier confusion after style transfer. Every row shows the classifier label distribution on sentences transferred to the target style (the row label). The off-diagonal elements show mis-classifications with intuitive domain similarities, such as (Lyrics, Poetry); (AAE, Tweets); (Joyce, Shakespeare).

feed style-specific tokens as input to a single model to control the output style (Johnson et al., 2017; Keskar et al., 2019). We adopt this strategy by feeding in the average RoBERTa-large [CLS ] vectors (by running the pretrained classifier on the training data) as style indicators to a single $f_{\text {inv }}^{i}$. We present results in Table 3 as - Mult. PP. This strategy is not as effective as training a different paraphrase model per style, primarily due to drop in ACC. We hypothesize this is due to a conflict between different style distributions in a single model.

\section{Towards Real-World Style Transfer}

All of our experiments and ablations thus far have been on the Shakespeare and Formality datasets, which contain just two styles each. To explore the ability of our system to perform style transfer between many diverse styles, we create the Corpus of Diverse Styles (CDS), a new non-parallel style transfer benchmark dataset with 11 diverse styles (15M tokens), and use it to evaluate STRAP.

Corpus of Diverse Styles: To create CDS, we obtain data (Table 5) from existing academic research datasets (Godfrey et al., 1992; Blodgett et al., 2016) and public APIs or online collections like Project Gutenberg (Hart, 1992). We choose styles that are easy for human readers to identify at a sentence level (e.g., Tweets or Biblical text), and the left side of Figure 2 confirms that machines also cluster CDS into eleven distinct styles. While prior benchmarks involve a transfer between two styles, CDS has 110 potential transfer directions. 


\begin{tabular}{|c|c|c|}
\hline Direction & Input & Output \\
\hline Tweet $\rightarrow$ Shak. & yall kissing before marriage? & And you kiss'd before your nuptial? \\
\hline Lyrics $\rightarrow$ AAE & It's a good thing you don't have bus fare & It's a good thing $\mathrm{u}$ aint gettin no ticket \\
\hline Tweet $\rightarrow$ Poetry & Its so disrespectful I hate itttttt & For'tis so rude, I have a sick regard, \\
\hline Shak. $\rightarrow$ Tweet & Need you my help? & Are yall okay? Like do you need my help?? \\
\hline AAE $\rightarrow$ Shak. & Aint got nooooo holiday spirit frfr & No spirit of this holiday, gentle man. \\
\hline Poetry $\rightarrow$ Bible & For the foul thief is just at your gate. & the wicked thief art at thy door. \\
\hline 1990. $\rightarrow$ Tweet & Now I can't talk to him about it. & I dont wanna talk to him abt that \\
\hline 1990. $\rightarrow$ Poetry & $\begin{array}{l}\text { I don't know why, but I sensed there was some- } \\
\text { thing wrong. }\end{array}$ & Something felt wrong; I knew not why, \\
\hline Tweet $\rightarrow$ Bible & $\begin{array}{l}\text { when ur going thru an episode and cant cope } \\
\text { anymore }\end{array}$ & $\begin{array}{l}\text { when thou art in an episode, and cannot stand it any } \\
\text { more; }\end{array}$ \\
\hline 1890. $\rightarrow$ AAE & $\begin{array}{l}\text { I was just thinking the same thing that you have } \\
\text { expressed. }\end{array}$ & u said the same thing i thought lol \\
\hline 1990. $\rightarrow 1810$ & $\begin{array}{l}\text { He was being terrorized into making a state- } \\
\text { ment by the same means as the other so-called } \\
\text { "witnesses." }\end{array}$ & $\begin{array}{l}\text { Terror had been employed in the same manner with the } \\
\text { other witnesses, to compel him to make a declaration. }\end{array}$ \\
\hline $\mathrm{AAE} \rightarrow$ Shak. & $\begin{array}{l}\text { If I got a dollar every time one of my friends } \\
\text { told me they hate me, I'd be rich }\end{array}$ & $\begin{array}{l}\text { I would have been rich, had I but a dollar for every } \\
\text { friend that hath said they hate me. }\end{array}$ \\
\hline Joyce $\rightarrow$ Bible & $\begin{array}{l}\text { I appeal for clemency in the name of the most } \\
\text { sacred word our vocal organs have ever been } \\
\text { called upon }\end{array}$ & $\begin{array}{l}\text { I beseech thee in the name of the most holy word which } \\
\text { is in our lips, forgive us our trespasses. }\end{array}$ \\
\hline
\end{tabular}

Table 4: Example outputs from STRAP trained on our CDS dataset (more generations in Appendix A.11).

\begin{tabular}{lr|lr}
\hline Style & Size & Style & Size \\
\hline Shakespeare & $27.5 \mathrm{~K}$ & Lyrics & $5.1 \mathrm{M}$ \\
James Joyce & $41.2 \mathrm{~K}$ & $1810-1830$ & $216.0 \mathrm{~K}$ \\
English Tweets & $5.2 \mathrm{M}$ & $1890-1910$ & $1.3 \mathrm{M}$ \\
AAE Tweets & $732.3 \mathrm{~K}$ & $1990-2010$ & $2.0 \mathrm{M}$ \\
Romantic Poetry & $29.8 \mathrm{~K}$ & Bible & $34.8 \mathrm{~K}$ \\
Switchboard & $148.8 \mathrm{~K}$ & & \\
\end{tabular}

Table 5: List of styles in our dataset along with the their total sizes. The year periods (like "1810-1830") refer to sentences from the Corpus of Historical American English (Davies, 2012). "AAE Tweets" refers to African American English Tweets corpus from Blodgett et al. (2016). "Switchboard" is a collection of conversational speech transcripts from Godfrey et al. (1992). Details of the collection and examples are in Appendix A.6.

We present dataset examples, details on collection and style similarity analysis in Appendix A.6.

\section{Diverse paraphrasing normalizes stylized text} With eleven styles, we can better validate the effectiveness of our diverse paraphraser at normalizing input sentences. After training an 11-way style classifier on CDS using RoBERTa-large, we observe an accuracy of $\mathbf{8 8 . 9 \%}$ on the original validation set. After paraphrasing the validation set with $f_{\text {para }}$, this classifier only correctly classifies 42.5\% sentences, indicating a significant decrease in recognizable stylistic features. Figure 2 further demonstrates this normalization effect. Finally, the magnitude of normalization is lower with the non-diverse paraphraser (from Section 5), with a smaller accuracy drop to $\mathbf{5 1 . 5 \%}$ after paraphras-

\begin{tabular}{lrrrr}
\hline \multicolumn{5}{c}{ Shakespeare $\leftrightarrow$ English Tweets, CDS } \\
\hline Model & ACC & SIM & FL & $J(\mathrm{~A}, \mathrm{~S}, \mathrm{~F})$ \\
\hline COPY & 0.1 & 100.0 & 69.2 & 0.0 \\
UNMT (2019) & 76.7 & 20.6 & 37.7 & 4.4 \\
DLSM $(2020)$ & 64.2 & 19.6 & 33.1 & 2.0 \\
\hline STRAP $(p=0.0)$ & 20.3 & 65.0 & 81.1 & 8.7 \\
STRAP $(p=0.6)$ & 31.1 & 58.1 & 75.0 & 10.8 \\
STRAP $(p=0.9)$ & 43.2 & 54.5 & 68.3 & $\mathbf{1 3 . 9}$ \\
\hline
\end{tabular}

Table 6: A controlled comparison between models on 2 styles from CDS using automatic evaluation. ACC is calculated using our 11-way CDS classifier and SIM is with input. STRAP greatly outperforms prior work.

ing; ${ }^{17}$ qualitatively, the diverse model exhibits more lexical swaps and syntactic diversity. ${ }^{18}$

Style Transfer on CDS: We measure STRAP's performance on CDS using Section 3's evaluation methodology. We sample $1 \mathrm{~K}$ sentences from each style and use STRAP to transfer these sentences to each of the 10 other styles. Despite having to deal with many more styles than before, our system achieves $\mathbf{4 8 . 4 \%}$ transfer accuracy (on a 11-way RoBERTa-large classifier), a paraphrase similarity score of $\mathbf{6 3 . 5}$, and $\mathbf{7 1 . 1 \%}$ fluent generations, yielding a $J$ (ACC,SIM,FL) score of 20.7. A break-

\footnotetext{
${ }^{17}$ Even if we retrain the classifiers on a paraphrased version of the training set (to model the distribution better), the performance is only $65.8 \%$ for the diverse model and $72.3 \%$ for the non-diverse model, indicating a loss in style signal.

${ }^{18}$ On average, the diverse model has $51 \%$ unigram $\mathrm{F} 1$ word overlap and $27 \%$ word shuffling measured by Kendall's $\tau_{\mathrm{B}}$, compared to $28 \%$ unigram F1 and $6 \%$ shuffling for the nondiverse model; Appendix A.7 has a style-wise breakdown.
} 


\begin{tabular}{lll}
\hline Direction & Input $\rightarrow$ Paraphrase $\rightarrow$ Output & Analysis \\
\hline Shak. $\rightarrow$ Bible & $\begin{array}{l}\text { Have you importuned him by any means? } \rightarrow \\
\text { did you ever try to import him? } \rightarrow \\
\text { hast thou ever tried to import him? }\end{array}$ & $\begin{array}{l}\text { Misunderstanding the word "importune" - } \\
\text { the model believes it refers to import rather } \\
\text { than harass / bother. }\end{array}$ \\
\hline 1990. $\rightarrow$ Tweet. & $\begin{array}{l}\text { The machine itself is made of little straws of carbon. } \rightarrow \\
\text { the machine is made of straw. } \rightarrow \\
\text { Machine made of straw. }\end{array}$ & $\begin{array}{l}\text { Dropping of important semantic words dur- } \\
\text { ing diverse paraphrasing ("carbon") signif- } \\
\text { icantly warps the meaning of sentences }\end{array}$ \\
\hline Swit. $\rightarrow$ Shak. & $\begin{array}{l}\text { well they offer classes out at uh Ray Hubbard } \rightarrow \\
\text { they're offering a course at Ray Hubbard's. } \rightarrow \\
\text { They do offer a course at the house of the Dukedom. }\end{array}$ & $\begin{array}{l}\text { Hallucination of tokens irrelevant to the } \\
\text { input ("house of the dukedom") to better } \\
\text { reflect style distribution. }\end{array}$ \\
\hline Tweet $\rightarrow$ Swit. & $\begin{array}{l}\text { Knoxville aint for me } \rightarrow \text { I'm not in Knoxville. } \rightarrow \\
\text { i don't know Knoxville }\end{array}$ & $\begin{array}{l}\text { Subtle modifications in semantics since the } \\
\text { models fail to understand their inputs. }\end{array}$ \\
\hline
\end{tabular}

Table 7: Representative examples showing the common failure modes of STRAP when evaluated on CDS.

down of style-specific performance is provided in Appendix A.8. An error analysis shows that the classifier misclassifies some generations as styles sharing properties with the target style (Figure 3).

Controlled comparisons: To ground our CDS results in prior work, we compare STRAP with baselines from Section 4.2. We sample equal number of training sentences from two challenging styles in CDS (Shakespeare, English Tweets) and train all three models (UNMT, DLSM, STRAP) on this subset of CDS. ${ }^{19}$ As seen in Table 6, STRAP greatly outperforms prior work, especially in SIM and FL. Qualitative inspection shows that baseline models often output arbitrary style-specific features, completely ignoring input semantics (explaining poor SIM but high ACC).

Qualitative Examples: Table 4 contains several outputs from STRAP; see Appendix A.11 for more examples. We also add more qualitative analysis of the common failures of our system in Table 7. Our model makes mistakes similar to contemporary text generation systems - poor understanding of rare words, dropping / modification of semantic content, hallucination to better reflect training distribution.

\section{Related Work}

Unsupervised style transfer is often modeled by disentangling style \& content using attribute classifiers (Hu et al., 2017; Shen et al., 2017), policy gradient training (Xu et al., 2018; Luo et al., 2019) or retrieval-based approaches (Li et al., 2018). Recently, backtranslation has emerged as a method to model semantic preservation (Prabhumoye et al.,

\footnotetext{
${ }^{19}$ We could not find an easy way to perform 11-way style transfer in the baseline models without significantly modifying their codebase / model due to the complex probabilistic formulation beyond 2 styles and separate modeling for each of the 110 directions.
}

2018), but this method can also warp semantics as seen in Subramanian et al. (2019); as such, we only use it to build our paraphraser's training data after heavy filtering. Our work relates to recent efforts that use Transformers in style transfer (Sudhakar et al., 2019; Dai et al., 2019). Closely related to our work is Gröndahl and Asokan (2019), who over-generate paraphrases using a complex handcrafted pipeline and filter them using proximity to a target style corpus. Instead, we automatically learn style-specific paraphrasers and do not need over-generation at inference. Relatedly, PreotiucPietro et al. (2016) present qualitative style transfer results with statistical MT paraphrasers. Other, less closely related work on control \& diversity in text generation is discussed in Appendix A.12.

\section{Conclusion}

In this work we model style transfer as a controlled paraphrase generation task and present a simple unsupervised style transfer method using diverse paraphrasing. We critique current style transfer evaluation using a survey of 23 papers and propose fixes to common shortcomings. Finally, we collect a new dataset containing $15 \mathrm{M}$ sentences from 11 diverse styles. Possible future work includes (1) exploring other applications of diverse paraphrasing, such as data augmentation; (2) performing style transfer at a paragraph level; (3) performing style transfer for styles unseen during training, using few exemplars provided during inference.

\section{Acknowledgements}

We thank the anonymous reviewers and area chair for their useful comments. We are grateful to $\mathrm{Su}$ Lin Blodgett, Katie Keith, Brendan O'Connor, Tu Vu, Shufan Wang, Nader Akoury, Rajarshi Das, Andrew Drozdov, Rico Angell, and the rest of the 
UMass NLP group for help at various stages of the project. Finally, we thank Arka Sadhu, Richard Pang, Kevin Gimpel, Graham Neubig, Diyi Yang, Shrimai Prabhumoye, Junxian He and Yonatan Belinkov for insightful discussions. This research was supported in part by a research gift from Adobe.

\section{References}

Eneko Agirre, Carmen Banea, Daniel Cer, Mona Diab, Aitor Gonzalez-Agirre, Rada Mihalcea, German Rigau, and Janyce Wiebe. 2016. SemEval-2016 task 1: Semantic textual similarity, monolingual and cross-lingual evaluation. In Proceedings of the 10th International Workshop on Semantic Evaluation (SemEval-2016).

Nader Akoury, Kalpesh Krishna, and Mohit Iyyer 2019. Syntactically supervised transformers for faster neural machine translation. In Proceedings of the Association for Computational Linguistics.

Martin Arjovsky, Soumith Chintala, and Léon Bottou. 2017. Wasserstein generative adversarial networks. In Proceedings of the International Conference of Machine Learning.

Dzmitry Bahdanau, Kyunghyun Cho, and Yoshua Bengio. 2014. Neural machine translation by jointly learning to align and translate. In Proceedings of the International Conference on Learning Representations.

Rahul Bhagat and Eduard Hovy. 2013. Squibs: What is a paraphrase? Computational Linguistics, 39(3):463-472.

Su Lin Blodgett, Lisa Green, and Brendan O'Connor. 2016. Demographic dialectal variation in social media: A case study of African-American English. In Proceedings of Empirical Methods in Natural Language Processing. Association for Computational Linguistics.

Ondřej Bojar, Ondřej Dušek, Tom Kocmi, Jindřich Libovický, Michal Novák, Martin Popel, Roman Sudarikov, and Dušan Variš. 2016. CzEng 1.6: Enlarged Czech-English Parallel Corpus with Processing Tools Dockered. In Text, Speech, and Dialogue: 19th International Conference, TSD 2016, number 9924 in Lecture Notes in Computer Science, pages 231-238, Cham / Heidelberg / New York / Dordrecht / London. Masaryk University, Springer International Publishing.

Samuel Bowman, Luke Vilnis, Oriol Vinyals, Andrew Dai, Rafal Jozefowicz, and Samy Bengio. 2016. Generating sentences from a continuous space. In Conference on Computational Natural Language Learning.

Chris Callison-Burch, Miles Osborne, and Philipp Koehn. 2006. Re-evaluating the role of Bleu in machine translation research. In Proceedings of the
European Chapter of the Association for Computational Linguistics.

Mingda Chen, Qingming Tang, Sam Wiseman, and Kevin Gimpel. 2019. Controllable paraphrase generation with a syntactic exemplar. In Proceedings of the Association for Computational Linguistics, Florence, Italy. Association for Computational Linguistics.

Ning Dai, Jianze Liang, Xipeng Qiu, and Xuanjing Huang. 2019. Style transformer: Unpaired text style transfer without disentangled latent representation. In Proceedings of the Association for Computational Linguistics.

Sumanth Dathathri, Andrea Madotto, Janice Lan, Jane Hung, Eric Frank, Piero Molino, Jason Yosinski, and Rosanne Liu. 2020. Plug and play language models: a simple approach to controlled text generation. In Proceedings of the International Conference on Learning Representations.

Mark Davies. 2012. Expanding horizons in historical linguistics with the 400-million word corpus of historical american english. Corpora.

Jacob Devlin, Ming-Wei Chang, Kenton Lee, and Kristina Toutanova. 2019. BERT: Pre-training of deep bidirectional transformers for language understanding. In Conference of the North American Chapter of the Association for Computational Linguistics, pages 4171-4186, Minneapolis, Minnesota. Association for Computational Linguistics.

Chrysanne DiMarco and Graeme Hirst. 1993. A computational theory of goal-directed style in syntax. Computational Linguistics, 19(3):451-499.

Penelope Eckert. 2008. Variation and the indexical field 1. Journal of sociolinguistics, 12(4).

Joseph L Fleiss. 1971. Measuring nominal scale agreement among many raters. Psychological bulletin, 76(5):378.

Markus Freitag, David Grangier, and Isaac Caswell 2020. Bleu might be guilty but references are not innocent. arXiv preprint arXiv:2004.06063.

Zhenxin Fu, Xiaoye Tan, Nanyun Peng, Dongyan Zhao, and Rui Yan. 2018. Style transfer in text: Exploration and evaluation. In Association for the $A d$ vancement of Artificial Intelligence.

John J Godfrey, Edward C Holliman, and Jane McDaniel. 1992. Switchboard: Telephone speech corpus for research and development. In IEEE International Conference on Acoustics, Speech, and Signal Processing.

Tanya Goyal and Greg Durrett. 2020. Neural syntactic preordering for controlled paraphrase generation. Proceedings of the Association for Computational Linguistics. 
Stephen J Green and Chrysanne DiMarco. 1993. Stylistic decision-making in natural language generation. In European Workshop on Trends in Natural Language Generation, pages 125-143. Springer.

Tommi Gröndahl and N Asokan. 2019. Effective writing style imitation via combinatorial paraphrasing. arXiv preprint arXiv:1905.13464.

Ankush Gupta, Arvind Agarwal, Prawaan Singh, and Piyush Rai. 2018. A deep generative framework for paraphrase generation. In Association for the Advancement of Artificial Intelligence.

Kelvin Guu, Tatsunori B Hashimoto, Yonatan Oren, and Percy Liang. 2018. Generating sentences by editing prototypes. Transactions of the Association for Computational Linguistics.

Michael Hart. 1992. The history and philosophy of Project Gutenberg. Project Gutenberg.

Junxian He, Xinyi Wang, Graham Neubig, and Taylor Berg-Kirkpatrick. 2020. A probabilistic formulation of unsupervised text style transfer. In Proceedings of the International Conference on Learning Representations.

George Heidorn. 2000. Intelligent writing assistance. Handbook of natural language processing, pages 181-207.

Peter Henderson, Riashat Islam, Philip Bachman, Joelle Pineau, Doina Precup, and David Meger. 2018. Deep reinforcement learning that matters. In Association for the Advancement of Artificial Intelligence.

Ari Holtzman, Jan Buys, Li Du, Maxwell Forbes, and Yejin Choi. 2020. The curious case of neural text degeneration. In Proceedings of the International Conference on Learning Representations.

Eduard Hovy. 1987. Generating natural language under pragmatic constraints. Journal of Pragmatics, 11(6):689-719.

Zhiting Hu, Zichao Yang, Xiaodan Liang, Ruslan Salakhutdinov, and Eric P Xing. 2017. Toward controlled generation of text. In Proceedings of the International Conference of Machine Learning.

Mohit Iyyer, John Wieting, Kevin Gimpel, and Luke Zettlemoyer. 2018. Adversarial example generation with syntactically controlled paraphrase networks. In Proceedings of the 2018 Conference of the North American Chapter of the Association for Computational Linguistics: Human Language Technologies, Volume 1 (Long Papers), pages 1875-1885, New Orleans, Louisiana. Association for Computational Linguistics.

Unnat Jain, Ziyu Zhang, and Alexander G Schwing. 2017. Creativity: Generating diverse questions using variational autoencoders. In Computer Vision and Pattern Recognition.
Melvin Johnson, Mike Schuster, Quoc V Le, Maxim Krikun, Yonghui Wu, Zhifeng Chen, Nikhil Thorat, Fernanda Viégas, Martin Wattenberg, Greg Corrado, et al. 2017. Google's multilingual neural machine translation system: Enabling zero-shot translation. Transactions of the Association for Computational Linguistics.

Jad Kabbara and Jackie Chi Kit Cheung. 2016. Stylistic transfer in natural language generation systems using recurrent neural networks. In Proceedings of the Workshop on Uphill Battles in Language Processing: Scaling Early Achievements to Robust Methods.

Daniel Kahneman. 2011. Thinking, fast and slow. Macmillan.

Jared Kaplan, Sam McCandlish, Tom Henighan, Tom B Brown, Benjamin Chess, Rewon Child, Scott Gray, Alec Radford, Jeffrey Wu, and Dario Amodei. 2020. Scaling laws for neural language models. arXiv preprint arXiv:2001.08361.

Divyansh Kaushik, Eduard Hovy, and Zachary C Lipton. 2020. Learning the difference that makes a difference with counterfactually-augmented data. In Proceedings of the International Conference on Learning Representations.

Maurice G Kendall. 1938. A new measure of rank correlation. Biometrika, 30(1/2):81-93.

Nitish Shirish Keskar, Bryan McCann, Lav R Varshney, Caiming Xiong, and Richard Socher. 2019. Ctrl: A conditional transformer language model for controllable generation. arXiv preprint arXiv:1909.05858.

Yoon Kim. 2014. Convolutional neural networks for sentence classification. In Proceedings of Empirical Methods in Natural Language Processing.

Diederik P Kingma and Jimmy Ba. 2015. Adam: A method for stochastic optimization. In Proceedings of the International Conference on Learning Representations.

Guillaume Klein, Yoon Kim, Yuntian Deng, Jean Senellart, and Alexander Rush. 2017. OpenNMT: Opensource toolkit for neural machine translation. In Proceedings of ACL 2017, System Demonstrations.

Stanley Kok and Chris Brockett. 2010. Hitting the right paraphrases in good time. In Conference of the North American Chapter of the Association for Computational Linguistics. Association for Computational Linguistics.

Ashutosh Kumar, Satwik Bhattamishra, Manik Bhandari, and Partha Talukdar. 2019. Submodular optimization-based diverse paraphrasing and its effectiveness in data augmentation. In Conference of the North American Chapter of the Association for Computational Linguistics. 
Guillaume Lample, Alexis Conneau, Ludovic Denoyer, and Marc' Aurelio Ranzato. 2018. Unsupervised machine translation using monolingual corpora only. In Proceedings of the International Conference on Learning Representations.

J Richard Landis and Gary G Koch. 1977. The measurement of observer agreement for categorical data. biometrics, pages 159-174.

Mike Lewis, Yinhan Liu, Naman Goyal, Marjan Ghazvininejad, Abdelrahman Mohamed, Omer Levy, Ves Stoyanov, and Luke Zettlemoyer 2019. BART: Denoising sequence-to-sequence pre-training for natural language generation, translation, and comprehension. arXiv preprint arXiv:1910.13461.

Jiwei Li, Will Monroe, and Dan Jurafsky. 2016. A simple, fast diverse decoding algorithm for neural generation. arXiv preprint arXiv:1611.08562.

Juncen Li, Robin Jia, He He, and Percy Liang. 2018. Delete, retrieve, generate: a simple approach to sentiment and style transfer. In Proceedings of the 2018 Conference of the North American Chapter of the Association for Computational Linguistics: Human Language Technologies, Volume 1 (Long Papers), pages 1865-1874, New Orleans, Louisiana. Association for Computational Linguistics.

Zhuohan Li, Eric Wallace, Sheng Shen, Kevin Lin, Kurt Keutzer, Dan Klein, and Joseph E Gonzalez. 2020. Train large, then compress: Rethinking model size for efficient training and inference of transformers. arXiv preprint arXiv:2002.11794.

Chia-Wei Liu, Ryan Lowe, Iulian Serban, Mike Noseworthy, Laurent Charlin, and Joelle Pineau. 2016. How NOT to evaluate your dialogue system: An empirical study of unsupervised evaluation metrics for dialogue response generation. In Proceedings of Empirical Methods in Natural Language Processing.

Yinhan Liu, Myle Ott, Naman Goyal, Jingfei Du, Mandar Joshi, Danqi Chen, Omer Levy, Mike Lewis, Luke Zettlemoyer, and Veselin Stoyanov. 2019 Roberta: A robustly optimized bert pretraining approach. arXiv preprint arXiv:1907.11692.

Lajanugen Logeswaran, Honglak Lee, and Samy Bengio. 2018. Content preserving text generation with attribute controls. In Proceedings of Advances in Neural Information Processing Systems.

Fuli Luo, Peng Li, Jie Zhou, Pengcheng Yang, Baobao Chang, Xu Sun, and Zhifang Sui. 2019. A dual reinforcement learning framework for unsupervised text style transfer. In International Joint Conference on Artificial Intelligence.

Thang Luong, Hieu Pham, and Christopher D. Manning. 2015. Effective approaches to attention-based neural machine translation. In Proceedings of Empirical Methods in Natural Language Processing.
Yun Ma, Yangbin Chen, Xudong Mao, and Qing Li. 2019. A syntax-aware approach for unsupervised text style transfer. OpenReview.

Aman Madaan, Amrith Setlur, Tanmay Parekh, Barnabas Poczos, Graham Neubig, Yiming Yang, Ruslan Salakhutdinov, Alan W Black, and Shrimai Prabhumoye. 2020. Politeness transfer: A tag and generate approach. In Proceedings of the Association for Computational Linguistics.

M. Meyerhoff. 2015. Introducing Sociolinguistics. Taylor \& Francis.

Remi Mir, Bjarke Felbo, Nick Obradovich, and Iyad Rahwan. 2019. Evaluating style transfer for text. In Conference of the North American Chapter of the Association for Computational Linguistics.

Shuyo Nakatani. 2010. Language detection library for java.

Jekaterina Novikova, Ondřej Dušek, Amanda Cercas Curry, and Verena Rieser. 2017. Why we need new evaluation metrics for NLG. In Proceedings of Empirical Methods in Natural Language Processing.

Myle Ott, Sergey Edunov, Alexei Baevski, Angela Fan, Sam Gross, Nathan Ng, David Grangier, and Michael Auli. 2019. fairseq: A fast, extensible toolkit for sequence modeling. In Proceedings of the 2019 Conference of the North American Chapter of the Association for Computational Linguistics (Demonstrations).

Richard Yuanzhe Pang. 2019. Towards actual (not operational) textual style transfer auto-evaluation. In Proceedings of the 5th Workshop on Noisy Usergenerated Text (W-NUT 2019).

Richard Yuanzhe Pang and Kevin Gimpel. 2019. Unsupervised evaluation metrics and learning criteria for non-parallel textual transfer. In Proceedings of the 3rd Workshop on Neural Generation and Translation.

Kishore Papineni, Salim Roukos, Todd Ward, and WeiJing Zhu. 2002. Bleu: a method for automatic evaluation of machine translation. In Proceedings of the Association for Computational Linguistics. Association for Computational Linguistics.

Sunghyun Park, Seung-won Hwang, Fuxiang Chen, Jaegul Choo, Jung-Woo Ha, Sunghun Kim, and Jinyeong Yim. 2019. Paraphrase diversification using counterfactual debiasing. In Association for the Advancement of Artificial Intelligence.

Hao Peng, Ankur Parikh, Manaal Faruqui, Bhuwan Dhingra, and Dipanjan Das. 2019. Text generation with exemplar-based adaptive decoding. In Conference of the North American Chapter of the Association for Computational Linguistics, Minneapolis, Minnesota. Association for Computational Linguistics. 
Shrimai Prabhumoye, Yulia Tsvetkov, Ruslan Salakhutdinov, and Alan W Black. 2018. Style transfer through back-translation. In Proceedings of the 56th Annual Meeting of the Association for Computational Linguistics (Volume 1: Long Papers), pages 866-876, Melbourne, Australia. Association for Computational Linguistics.

Daniel Preotiuc-Pietro, Wei Xu, and Lyle Ungar. 2016. Discovering user attribute stylistic differences via paraphrasing. In Association for the Advancement of Artificial Intelligence.

Alec Radford, Jeffrey Wu, Rewon Child, David Luan, Dario Amodei, and Ilya Sutskever. 2019. Language models are unsupervised multitask learners. OpenAI Blog, 1(8).

Colin Raffel, Noam Shazeer, Adam Roberts, Katherine Lee, Sharan Narang, Michael Matena, Yanqi Zhou, Wei Li, and Peter J Liu. 2019. Exploring the limits of transfer learning with a unified text-to-text transformer. arXiv preprint arXiv:1910.10683.

Pranav Rajpurkar, Jian Zhang, Konstantin Lopyrev, and Percy Liang. 2016. SQuAD: 100,000+ questions for machine comprehension of text. In Proceedings of Empirical Methods in Natural Language Processing.

Sudha Rao and Joel Tetreault. 2018. Dear sir or madam, may I introduce the GYAFC dataset: Corpus, benchmarks and metrics for formality style transfer. In Conference of the North American Chapter of the Association for Computational Linguistics.

Cicero Nogueira dos Santos, Igor Melnyk, and Inkit Padhi. 2018. Fighting offensive language on social media with unsupervised text style transfer. In Proceedings of the Association for Computational Linguistics.

Thibault Sellam, Dipanjan Das, and Ankur Parikh. 2020. BLEURT: Learning robust metrics for text generation. In Proceedings of the Association for Computational Linguistics.

Rico Sennrich, Barry Haddow, and Alexandra Birch. 2016. Improving neural machine translation models with monolingual data. In Proceedings of the 54th Annual Meeting of the Association for Computational Linguistics (Volume 1: Long Papers), pages 86-96, Berlin, Germany. Association for Computational Linguistics.

Tianxiao Shen, Tao Lei, Regina Barzilay, and Tommi Jaakkola. 2017. Style transfer from non-parallel text by cross-alignment. In Advances in neural information processing systems, pages 6830-6841.

Rakshith Shetty, Bernt Schiele, and Mario Fritz. 2018. A4nt: author attribute anonymity by adversarial training of neural machine translation. In 27th $\{$ USENIX $\}$ Security Symposium ( $\{$ USENIX $\}$ Security 18), pages 1633-1650.
Sandeep Subramanian, Guillaume Lample, Eric Michael Smith, Ludovic Denoyer, Marc'Aurelio Ranzato, and Y-Lan Boureau. 2019. Multiple-attribute text style transfer. In Proceedings of the International Conference on Learning Representations.

Akhilesh Sudhakar, Bhargav Upadhyay, and Arjun Maheswaran. 2019. "transforming" delete, retrieve, generate approach for controlled text style transfer. In Proceedings of Empirical Methods in Natural Language Processing.

Ilya Sutskever, Oriol Vinyals, and Quoc V Le. 2014. Sequence to sequence learning with neural networks. In Proceedings of Advances in Neural Information Processing Systems, pages 3104-3112.

Bakhtiyar Syed, Gaurav Verma, Balaji Vasan Srinivasan, Vasudeva Varma, et al. 2020. Adapting language models for non-parallel author-stylized rewriting. In Association for the Advancement of Artificial Intelligence.

Alexey Tikhonov, Viacheslav Shibaev, Aleksander Nagaev, Aigul Nugmanova, and Ivan P Yamshchikov. 2019. Style transfer for texts: Retrain, report errors, compare with rewrites. In Proceedings of Empirical Methods in Natural Language Processing.

Ashish Vaswani, Noam Shazeer, Niki Parmar, Jakob Uszkoreit, Llion Jones, Aidan N Gomez, Łukasz Kaiser, and Illia Polosukhin. 2017. Attention is all you need. In Proceedings of Advances in Neural Information Processing Systems, pages 5998-6008.

Ashwin K Vijayakumar, Michael Cogswell, Ramprasaath R Selvaraju, Qing Sun, Stefan Lee, David Crandall, and Dhruv Batra. 2018. Diverse beam search for improved description of complex scenes. In Association for the Advancement of Artificial Intelligence.

Pascal Vincent, Hugo Larochelle, Yoshua Bengio, and Pierre-Antoine Manzagol. 2008. Extracting and composing robust features with denoising autoencoders. In Proceedings of the International Conference of Machine Learning, pages 1096-1103.

Alex Wang, Kyunghyun Cho, and Mike Lewis. 2020. Asking and answering questions to evaluate the factual consistency of summaries. In Proceedings of the Association for Computational Linguistics.

Alex Warstadt, Amanpreet Singh, and Samuel R Bowman. 2019. Neural network acceptability judgments. Transactions of the Association for Computational Linguistics.

John Wieting, Taylor Berg-Kirkpatrick, Kevin Gimpel, and Graham Neubig. 2019. Beyond BLEU:training neural machine translation with semantic similarity. In Proceedings of the Association for Computational Linguistics. 
John Wieting and Kevin Gimpel. 2018. ParaNMT50M: Pushing the limits of paraphrastic sentence embeddings with millions of machine translations. In Proceedings of the 56th Annual Meeting of the Association for Computational Linguistics (Volume 1: Long Papers), pages 451-462, Melbourne, Australia. Association for Computational Linguistics.

Sam Wiseman, Stuart Shieber, and Alexander Rush. 2018. Learning neural templates for text generation. In Proceedings of Empirical Methods in Natural Language Processing.

Thomas Wolf, Lysandre Debut, Victor Sanh, Julien Chaumond, Clement Delangue, Anthony Moi, Pierric Cistac, Tim Rault, R'emi Louf, Morgan Funtowicz, and Jamie Brew. 2019. HuggingFace's Transformers: State-of-the-art natural language processing. ArXiv, abs/1910.03771.

Thomas Wolf, Victor Sanh, Julien Chaumond, and Clement Delangue. 2018. Transfertransfo: A transfer learning approach for neural network based conversational agents. In NeurIPS CAI Workshop.

Qizhe Xie, Zihang Dai, Eduard Hovy, Minh-Thang Luong, and Quoc V Le. 2019. Unsupervised data augmentation for consistency training. arXiv preprint arXiv:1904.12848.

Jingjing Xu, Xu Sun, Qi Zeng, Xiaodong Zhang, Xuancheng Ren, Houfeng Wang, and Wenjie Li. 2018. Unpaired sentiment-to-sentiment translation: A cycled reinforcement learning approach. In Proceedings of the Association for Computational Linguistics.

Wei Xu, Chris Callison-Burch, and Courtney Napoles. 2015. Problems in current text simplification research: New data can help. Transactions of the Association for Computational Linguistics, 3:283-297.

Wei Xu, Alan Ritter, Bill Dolan, Ralph Grishman, and Colin Cherry. 2012. Paraphrasing for style. In Proceedings of International Conference on Computational Linguistics.

Zichao Yang, Zhiting Hu, Chris Dyer, Eric P Xing, and Taylor Berg-Kirkpatrick. 2018. Unsupervised text style transfer using language models as discriminators. In Proceedings of Advances in Neural Information Processing Systems.

Kuo-Hao Zeng, Mohammad Shoeybi, and Ming-Yu Liu. 2020. Style example-guided text generation using generative adversarial transformers. arXiv preprint arXiv:2003.00674.

Zhirui Zhang, Shuo Ren, Shujie Liu, Jianyong Wang, Peng Chen, Mu Li, Ming Zhou, and Enhong Chen. 2018. Style transfer as unsupervised machine translation. arXiv preprint arXiv:1808.07894.
Daniel M Ziegler, Nisan Stiennon, Jeffrey Wu, Tom B Brown, Alec Radford, Dario Amodei, Paul Christiano, and Geoffrey Irving. 2019. Fine-tuning language models from human preferences. arXiv preprint arXiv:1909.08593. 


\section{A Appendices for "Reformulating Unsupervised Style Transfer as Paraphrase Generation"}

\section{A.1 PARANMT-50M Filtering Details}

We train our paraphrase model in a seq2seq fashion using the PARANMT-50M corpus (Wieting and Gimpel, 2018), which was constructed by backtranslating (Sennrich et al., 2016) the Czech side of the CzEng parallel corpus (Bojar et al., 2016). This corpus is large and noisy and we aggressively filter it to encourage content preservation and diversity maximization. We use the following filtering,

Content Filtering: We remove all sentence pairs which score lower than 0.5 on a strong paraphrase similarity model from Wieting et al. (2019). ${ }^{20} \mathrm{We}$ filter sentence pairs by length, allowing a maximum length difference of 5 words between paired sentences. Finally, we remove very short and long sentences by only keeping sentence pairs with an average token length between 7 and 25 .

Lexical Diversity Filtering: We only preserve backtranslated pairs with sufficient unigram distribution difference. We filter all pairs where more than $50 \%$ of words in the backtranslated sentence can be found in the source sentence. This is computed using the SQuAD evaluation scripts (Rajpurkar et al., 2016). Additionally, we remove sentences with more than $70 \%$ trigram overlap.

Syntactic Diversity Filtering: We discard all paraphrases which have a similar word ordering. We compare the relative ordering of the words shared between the input and backtranslated sentence by measuring the Kendall tau distance (Kendall, 1938) or the "bubble-sort" distance. We keep all backtranslated pairs which are at least $50 \%$ shuffled. ${ }^{21}$

LangID Filtering: Finally, we discard all sentences where both the input and backtranslated sentence are classified as non-English using langdetect. ${ }^{22}$

Effect of each filter: We adopt a pipelined approach to filtering. The PARANMT-50M corpus size after each stage of filtering is shown in Table 8.

\footnotetext{
${ }^{20}$ We use the SIM model from Wieting et al. (2019), which achieves a strong performance on the SemEval semantic text similarity (STS) benchmarks (Agirre et al., 2016)

${ }^{21} \mathrm{An}$ identical ordering of words is $0 \%$ shuffled whereas a reverse ordering is $100 \%$ shuffled.

${ }^{22}$ This is using the Python port of Nakatani (2010), https : //github.com/Mimino666/langdetect.
}

\begin{tabular}{llr}
\hline & Filter Stage & Corpus Size \\
\hline 0. & Original & $51.41 \mathrm{M}$ \\
1. & Content Similarity & $30.49 \mathrm{M}$ \\
2. & Trigram Diversity & $9.03 \mathrm{M}$ \\
3. & Unigram Diversity & $1.96 \mathrm{M}$ \\
4. & Kendall-Tau Diversity & $112.01 \mathrm{~K}$ \\
5. & Length Difference & $82.64 \mathrm{~K}$ \\
6. & LangID & $74.55 \mathrm{~K}$ \\
\hline
\end{tabular}

Table 8: Steps of filtering conducted on PARANMT$50 \mathrm{M}$ along with its effect on corpus size.

\section{A.2 Generative Model Details}

This section provides details of our seq2seq model used for both paraphrase model and style-specific inverse paraphrase model. Recent work (Radford et al., 2019) has shown that GPT2, a massive transformer trained on a large corpus of unlabeled text using the language modeling objective, is very effective in performing more human-like text generation. We leverage the publicly available GPT2-large checkpoints by finetuning it on our custom datasets with a small learning rate. However, GPT2 is an unconditional language model having only a decoder network, and traditional seq2seq setups use separate encoder and decoder neural network (Sutskever et al., 2014) with attention (Bahdanau et al., 2014). To avoid training an encoder network from scratch, we use the encoder-free seq2seq modeling approach described in Wolf et al. (2018). where both input and output sequences are fed to the decoder network separated with a special token, and use separate segment embeddings. Our model is implemented using the transformers library ${ }^{23}$ (Wolf et al., 2019). We use encoder-free seq2seq modeling (Wolf et al., 2018) which feeds the input into the decoder neural network, separating it with segment embeddings. We fine-tune GPT2-large to perform encoder-free seq2seq modeling.

Architecture: Let $\mathbf{x}=\left(x_{1}, \ldots, x_{n}\right)$ represent the tokens in the input sequence and let $\mathbf{y}=$ $\left(y_{b o s}, y_{1}, \ldots, y_{m}, y_{\text {eos }}\right)$ represent the tokens of the output sequence, where $y_{b o s}$ and $y_{\text {eos }}$ corresponds to special beginning and end of sentence tokens. We feed the sequence $\left(x_{1}, \ldots, x_{n}, y_{b o s}, y_{1}, \ldots, y_{m}\right)$ as input to GPT2 and train it on the next-word prediction objective for the tokens $y_{1}, \ldots, y_{m}, y_{e o s}$

\footnotetext{
${ }^{23}$ https://github. com/huggingface/ transformers
} 
using the cross-entropy loss. During inference, the sequence $\left(x_{1}, \ldots, x_{n}, y_{b o s}\right)$ is fed as input and the tokens are generated in an autoregressive manner (Vaswani et al., 2017) until $y_{\text {eos }}$ is generated.

Every token in $\mathbf{x}$ and $\mathbf{y}$ is passed through a shared input embedding layer to obtain a vector representation of every token. To encode positional and segment information, learnable positional and segment embeddings are added to the input embedding consistent with the GPT2 architecture. Segment embeddings are used to denote whether a token belongs to sequence $\mathrm{x}$ or $\mathrm{y}$.

Other seq2seq alternatives: Note that our unsupervised style transfer algorithm is agnostic to the specific choice of seq2seq modeling. We wanted to perform transfer learning from massive left-to-right language models like GPT2, and found the encoder-free seq2seq approach simple and effective. Future work includes finetuning more recent models like T5 (Raffel et al., 2019) or BART (Lewis et al., 2019). These models use the standard seq2seq setup of separate encoder / decoder networks and pretrain them jointly using denoising autoencoding objectives based on language modeling.

Hyperparameter Details: We finetune GPT2large using NVIDIA TESLA M40 GPUs for 2 epochs using early stopping based on validation set perplexity. The models are finetuned using a small learning rate of $5 \mathrm{e}-5$ and converge to a good solution fairly quickly as noticed by recent work ( $\mathrm{Li}$ et al., 2020; Kaplan et al., 2020). Specifically, each experiment completed within a day of training on a single GPU, and many experiments with small datasets took a lot less time. We use a minibatch size of 10 sentence pairs and truncate sequences which are longer than 50 subwords in the input or output space. We use the Adam optimizer (Kingma and $\mathrm{Ba}, 2015$ ) with the weight decay fix and using a linear learning rate decay schedule, as implemented in the transformers library. Finally, we leftpad the input sequence to get a total input length of 50 subwords and right-pad output sequence to get a total output length of 50 subwords. This special batching is necessary to use minibatches during inference time. Special symbols are used to pad the sequences and they are not considered in the cross-entropy loss. Our model has 774M trainable parameters, identical to the original GPT2-large.

\section{A.3 Classifier Model Details}

We fine-tune RoBERTa-large to build our classifier, using the official implementation in fairseq. We use a learning rate of $1 \mathrm{e}-5$ for all experiments with a minibatch size of 32. All models were trained on a single NVIDIA RTX 2080ti GPU, with gradient accumulation to allow larger batch sizes. We train models for 10 epochs and use early stopping on the validation split accuracy. We use the Adam optimizer (Kingma and Ba, 2015) with modifications suggested in the RoBERTa paper (Liu et al., 2019). Consistent with the suggested hyperparameters, we use a learning rate warm-up for the first $6 \%$ of the updates and then decay the learning rate.

\section{A.4 OpenNMT Model Details}

We train sequence-to-sequence models with attention based on LSTMs using OpenNMT (Klein et al., 2017) using their PyTorch port. ${ }^{24} \mathrm{We}$ mostly used the default hyperparameter settings of OpenNMT-py. The only hyperparameter we modified was the learning rate schedule, since our datasets were small and overfit quickly. For the paraphrase model, we started decay after 11000 steps and halved the learning rate every 1000 steps. For Shakespeare, we started the decay after 3000 steps and halved the learning rate every 500 steps. For Formality, we started the decay after 6000 steps and halved the learning rate every 1000 steps. These modifications only slightly improved validation perplexity (by 3-4 points in each case).

We used early stopping on validation perplexity and checkpoint the model every 500 optimization steps. The other hyperparameters are the default OpenNMT-py settings - SGD optimization using learning rate 1.0, LSTM seq2seq model with global attention (Luong et al., 2015), 500 hidden units and embedding dimensions and 2 layers each in the encoder and decoder.

\section{A.5 More Comparisons with Prior Work}

Please refer to Table 12 for an equivalent of Table 1 using BLEU scores.

We present more comparisons with prior work in Table 13. We use the generated outputs for the Formality test set available in the public repository of Luo et al. (2019) (including outputs from the algorithms described in Prabhumoye et al., 2018 and Li et al., 2018) and run them on our evaluation pipeline. We compare the results

\footnotetext{
${ }^{24}$ https: / / github.com/OpenNMT / OpenNMT-py
} 
with our formality transfer model used in Table 1 and Table 2. We note significant performance improvements, especially in the fluency of the generated text. Note that there is a domain shift for our model, since we trained our model using the splits of He et al. (2020) which use the Entertainment \& Music splits of the Formality corpus. The outputs in the repository of Luo et al. (2019) use the Family \& Relationships split. It is unclear in the paper of Luo et al. (2019) whether the models were trained on the Family \& Relationships training split or not.

Other Comparisons: We tried to compare against other recent work in style transfer based on Transformers, such as Dai et al. (2019) and Sudhakar et al. (2019). Both papers do not evaluate their models on the datasets we use (Shakespeare and Formality), where parallel sentences preserve semantics.

The only datasets used in Dai et al. (2019) were sentiment transfer benchmarks, which modify semantic properties of the sentence. We attempted to train the models in Dai et al. (2019) using their codebase on the Shakespeare dataset, but faced three major issues 1) missing number of epochs / iterations. The early stopping criteria is not implemented or specified, and metrics were being computed on the test set every 25 training iterations, which is invalid practice for choosing the optimal checkpoint; 2) specificity of the codebase to the Yelp sentiment transfer dataset in terms of maximum sequence length and evaluation, making it non-trivial to use for any other dataset; 3) despite our best efforts we could not get the model to converge to a good minima which would produce fluent text (besides word-by-word copying) when trained on the Shakespeare dataset.

Similarly, the datasets used in Sudhakar et al (2019) modify semantic properties (sentiment, political slant etc.). On running their codebase on the Shakespeare dataset using the default hyperparameters, we achieved a poor performance of $53.1 \%$ ACC, 55.2 SIM and $56.5 \% \mathrm{FL}$, aggregating to a $J(\mathrm{~A}, \mathrm{~S}, \mathrm{~F})$ score of 18.4. Similarly on the Formality dataset, performance was poor with $41.7 \% \mathrm{ACC}$, 67.8 SIM and $67.7 \%$ FL, aggregating to $J(\mathrm{~A}, \mathrm{~S}, \mathrm{~F})$ score of 18.1. A qualitatively inspection showed very little abstraction and nearly word-by-word copying from the input (due to the delete \& generate nature of the approach), which explains the higher SIM score but lower ACC score (just like COPY baseline in Table 1). Fluency was low despite GPT pretraining, perhaps due to the token deletion step in the algorithm.

\section{A.6 Details of our Dataset, CDS}

We provide details of our sources, the sizes of individual style corpora and examples from our new benchmark dataset CDS in Table 14. We individually preprocessed each corpus to remove very short and long sentences, boilerplate text (common in Project Gutenberg articles) and section headings. We have added some representative examples from each style in Table 14. More representative examples (along with our entire dataset) will be provided in the project page http://style.cs.umass.edu.

Style Similarity: In Figure 4 we plot the cosine similarity between styles using the averaged [CLS ] vector of the trained RoBERTa-large classifier (inference over validation set). The offdiagonal elements show intuitive domain similarities, such as (Lyrics, Poetry); (AAE, Tweets); (Joyce, Shakespeare) or among classes from the Corpus of Historical American English.

\section{A.7 Diverse Paraphrasing on CDS}

We compare the quality and diversity of the paraphrases generated by our diverse and non-diverse paraphrasers on our dataset CDS in Table 16. Note that this is the pseudo parallel training data for the inverse paraphrase model (described in Section 2.1 and Section 2.4) and not the actual style transferred sentences. Overall, the diverse paraphraser achieves high diversity, with 51\% unigram change and $27 \%$ word shuffling, ${ }^{25}$ compared to $28 \%$ unigram and $6 \%$ shuffling for non-diverse paraphraser, while maintaining good semantic similarity (SIM= 72.5 vs 83.9 for non-diverse) even in complex stylistic settings.

\section{A.8 Style Transfer Performance on CDS}

We provide a detailed breakdown of performance in different styles of CDS in Table 15. For each of the 11 target styles, we style transferred 1,000 sentences from every other style and jointly evaluated the 10,000 generations. Some styles are more successfully transferred than others, such as Switchboard, Lyrics and James Joyce. While wearing the

\footnotetext{
${ }^{25}$ The "unigram change" and "word shuffling" refer to the unigram F1 word overlap and Kendall's $\tau_{\mathrm{B}}$ scores.
} 


\section{Original Sentences Cosine Similarity}

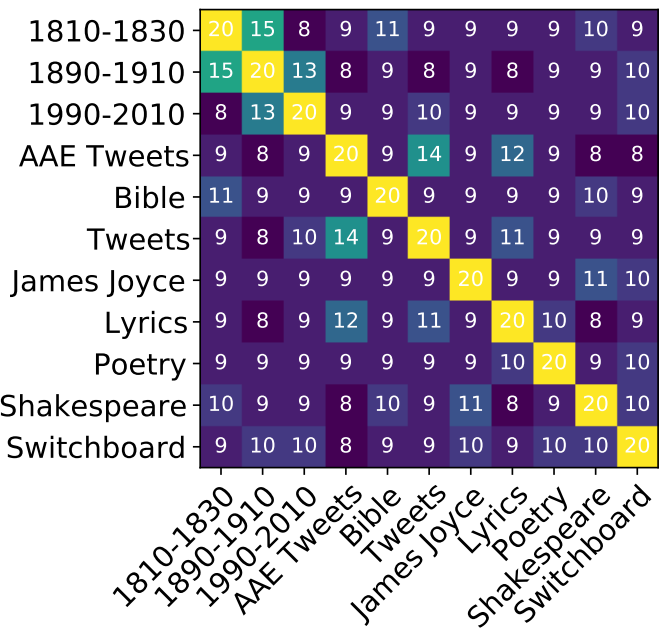

Figure 4: Cosine similarities between styles in CDS using the [CLS] vectors of the RoBERTa-large classifier (normalized to $[0,20]$ ). The off-diagonal elements show intuitive domain similarities, such as (Lyrics, Poetry); (AAE, Tweets); (Joyce, Shakespeare) or among classes from the COHA corpus.

$p$ value for nucleus sampling, we notice a trend similar to the Nucleus sampling trades off ACC for SIM experiment in Section 5. Increasing the $p$ value improves ACC at the cost of SIM. However unlike the Shakespeare and Formality dataset, we find $p=0.6$ the optimal value for the best ACCSIM tradeoff.

Note that Fluency scores on this dataset could be misleading since even the original sentences from some styles are often classified as disfluent (Orig. FL). Qualitatively, this seems to happen for styles with rich lexical and syntactic diversity (like Romantic Poetry, James Joyce). These styles tend to be out-of-distribution for the fluency classifier trained on the CoLA dataset (Warstadt et al., 2019).

\section{A.9 A Survey of Evaluation Methods}

We present a detailed breakdown of evaluation metrics used in prior work in Table 10 and the implementations of the metrics in Table 11. Notably, only 3 out of 23 prior works use an absolute sentence-level aggregation evaluation. Other works either perform "overall A/B" testing, flawed corpuslevel aggregation or don't perform any aggregation at all. Note that while "overall A/B" testing cannot be gamed like corpus-aggregation, it has a few issues - (1) it is a relative evaluation and does not provided an absolute performance score for future reference; (2) "A/B" testing requires human evalu- ation, which is expensive and noisy; (3) evaluating overall performance will require human annotators to be familiar with the styles and style transfer task setup; (4) Kahneman (2011) has shown that asking humans to give a single number for "overall score" is biased when compared to an aggregation of independent scores on different metrics. Luckily, the sentence-level aggregation in Li et al. (2018) does the latter and is the closest equivalent to our proposed $J(\cdot)$ metric.

\section{A.10 Details on Human Evaluation}

We conduct experiments of Amazon Mechanical Turk, annotating the paraphrase similarity of 150 sentences with 3 annotators each. We report the label chosen by two or more annotators, and collect additional annotations in the case of total disagreement. We pay workers 5 cents per sentence pair ( $\$ 10-15 / \mathrm{hr})$. We only hire workers from USA, UK and Australia with a 95\% or higher approval rating and at least 1000 approved HITs. Sentences where the input was exactly copied (after lower-casing and removing punctuation) are automatically assigned the option $\mathbf{2}$ paraphrase and grammatical. Even though these sentences are clearly not style transferred, we expect them to be penalized in $J$ (ACC,SIM,FL) by poor ACC. We found that every experiment had a Fleiss kappa (Fleiss, 1971) of at least 0.13 and up to 0.45 (slight to moderate agreement according to (Landis and Koch, 1977)). A qualitative inspection showed that crowdworkers found it easier to judge sentence pairs in the Formality dataset than Shakespeare, presumably due to greater familiarity with modern English. We also note that crowdworkers had higher agreement for sentences which were clearly not paraphrases (like the UNMT / DLSM generations on the Formality dataset).

Calculating Metrics in Table 2: To calculate SIM, we count the percentage of sentences which humans assigned a label 1 (ungrammatical paraphrase) or 2 (grammatical paraphrase). This is used as a binary value to calculate $J$ (ACC, SIM). To calculate $J$ (ACC, SIM, FL), we count sentences which are correctly classified as well as humans assigned a label of $\mathbf{2}$ (grammatical paraphrase). We cannot calculate FL alone using the popular 3-way evaluation, since the fluent sentences which are not paraphrases are not recorded. 


\section{A.11 More Example Generations}

More examples are provided in Table 9. All of our style transferred outputs on CDS will be available in the project page of this work, http://style.cs.umass.edu.

\section{A.12 More Related Work}

Our inverse paraphrase model is a style-controlled text generator which automatically learns lexical and syntactic properties prevalent in the style's corpus. Explicit syntactically-controlled text generation has been studied previously using labels such as constituency parse templates (Iyyer et al., 2018; Akoury et al., 2019) or learned discrete latent templates (Wiseman et al., 2018). Syntax can also be controlled using an exemplar sentence (Chen et al., 2019; Guu et al., 2018; Peng et al., 2019). While style transfer requires the underlying content to be provided as input, another direction explores attribute-controlled unconditional text generation (Dathathri et al., 2020; Keskar et al., 2019; Zeng et al., 2020; Ziegler et al., 2019).

Diversity in text generation is often encouraged during inference time via heuristic modifications to beam search (Li et al., 2016; Vijayakumar et al., 2018), nucleus sampling (Holtzman et al., 2020) or submodular optimization (Kumar et al., 2019); in contrast, we simply filter our training data to increase diversity. Other algorithms learn to condition generation on latent variables during training (Bowman et al., 2016), which are sampled from at inference time to encourage diversity (Jain et al., 2017; Gupta et al., 2018; Park et al., 2019). Relatedly, Goyal and Durrett (2020) promote syntactic diversity of paraphrases by conditioning over possible syntactic rearrangements of the input. 


\begin{tabular}{|c|c|c|}
\hline Direction & Input & Output \\
\hline 1810. $\rightarrow$ Swit. & $\begin{array}{l}\text { She forgot her gold pieces lying on the table in } \\
\text { front of her. }\end{array}$ & and uh she forgot the gold pieces on the desk \\
\hline 1810. $\rightarrow$ AAE & This story is not well devised, my lord. & Lord this story ain't no good \\
\hline Poetry $\rightarrow$ Swit. & Why should our young Endymion pine away! & $\begin{array}{l}\text { i don't know why we should have such a letdown for } \\
\text { our young Endymion }\end{array}$ \\
\hline Tweet $\rightarrow$ Shak. & This is an insult to the Munster's. & Tis an affront to the Munster. \\
\hline Swit. $\rightarrow 1810$. & $\begin{array}{l}\text { and my lawn is very green and my flowers are } \\
\text { still blooming }\end{array}$ & I have plenty of green turf and flowers here. \\
\hline 1890. $\rightarrow$ AAE & I want no pursuit when I do my work. & I dont wanna chase when im at work \\
\hline 1990. $\rightarrow$ Joyce & "You're scaring me," Clara says & 'Tis scare me, says Clara. \\
\hline Tweet $\rightarrow$ Bible & $\begin{array}{l}\text { Can we build AI without losing control over } \\
\text { it? }\end{array}$ & Can a man build a computer without losing his power? \\
\hline $\mathrm{AAE} \rightarrow 1810$ & I Use To Think They Were Ugly. & I thought them odious-and hateful. \\
\hline Bible $\rightarrow 1990$. & hath thy soul lothed Zion? & Is Zion a place where you have your soul? \\
\hline Bible $\rightarrow$ Tweet & $\begin{array}{l}\text { This persuasion cometh not of him that calleth } \\
\text { you. }\end{array}$ & Its not him who comes from. Its who you call \\
\hline Swit. $\rightarrow$ AAE & um yeah just a second & yea just a lil bit \\
\hline Joyce $\rightarrow$ Shak. & Smell of burn. & This is the smoke of a burning. \\
\hline 1990. $\rightarrow$ Joyce & The Second World War, son," he answered. & SECOND WORLD WAR, son, says he. \\
\hline 1990. $\rightarrow$ AAE & no, I implore you not to consider this option. & Naw I asked u not to think bout that option \\
\hline Tweet $\rightarrow$ Joyce & i wanted to sleep but now i cant & sleep is my preferred repose. \\
\hline 1990. $\rightarrow 1810$ & She ninety-three and she can't hear too good. & She is 93 years of age and cannot hear well yet. \\
\hline $\mathrm{AAE} \rightarrow$ Poetry & y'all play? we spray & And weel weel, we'll spray a' that. \\
\hline Joyce $\rightarrow$ Poetry & Now, don't tell a big fib! & And say not so big a lie! \\
\hline 1810. $\rightarrow$ Bible & without first veiling that, I could not sin. & And I could not sin without veil. \\
\hline 1890. $\rightarrow$ Shak. & $\begin{array}{l}\text { When did we ever find Gaston de Marignac } \\
\text { objectionable? }\end{array}$ & $\begin{array}{l}\text { When was 't that we found Gaston de Marignac's foul } \\
\text { faults? }\end{array}$ \\
\hline Shak. $\rightarrow 1990$ & $\mathrm{O}$, much I fear some ill unlucky thing. & I was afraid of a lot of misadventures. \\
\hline Shak. $\rightarrow 1810$. & By Jesu, a very good blade! & The very knife of heaven, Jesu! \\
\hline Lyrics $\rightarrow 1890$ & For a thing to explode & Oh, oh, oh, for something to blow up! \\
\hline Lyrics $\rightarrow$ Bible & You are the sun that lights my world & $\begin{array}{l}\text { Thou art the sun that lighteth mine earth: thou art my } \\
\text { righteousness. }\end{array}$ \\
\hline Lyrics $\rightarrow$ Joyce & But now it shouldn't have to be this way & But oughtn't to be like that. \\
\hline Swit. $\rightarrow 1810$ & oh that & deor littlo \\
\hline Swit. $\rightarrow$ Lyrics & you know Karen i wonder if we are recording & Karen, I wonder if we're tapein' \\
\hline Tweet $\rightarrow$ Bible & Ok will get back to you ASAP! & And he said, All right; I will return. \\
\hline Tweet $\rightarrow$ Shak. & Movies are always funnier in the cinema. & It always is more humorous in the theatre. \\
\hline Lyrics $\rightarrow$ Poetry & You know I still fear the bomb & I was yet a-fear'd at the bomb, \\
\hline Joyce $\rightarrow 1890$. & Just missed that by a hair. & It was a hair-spitting thing I missed. \\
\hline Joyce $\rightarrow 1810$. & Me seemeth a dragon man. & Hark ye, I'm a dragon man, Me. \\
\hline Poetry $\rightarrow$ Bible & Of deities or mortals, or of both, & $\begin{array}{l}\text { Of the gods, and of the people, or of the both of them } \\
\text { together. }\end{array}$ \\
\hline Poetry $\rightarrow$ Lyrics & Make & Gonna be a lot of people mourning! \\
\hline Poetry $\rightarrow$ Bible & My helpless lambs, I trust them wi' him. & Surely he will feed my lambs in safety. \\
\hline 1890. $\rightarrow$ Poetry & $\begin{array}{l}\text { Stark was sentenced to ten years' imprison- } \\
\text { ment, Gibbon to five. }\end{array}$ & Stark was sentence'd ten yeares, Gibbon five; \\
\hline 1890. $\rightarrow$ Swit. & I want no pursuit when I do my work. & i don't want to chase after when i'm at work \\
\hline Tweet $\rightarrow$ Bible & The way this is set up has me dying & This death cometh because of this. \\
\hline Tweet $\rightarrow$ Swit. & $\begin{array}{l}\text { Yay! Ill order them then! I dont have patience } \\
\text { to wait on them! }\end{array}$ & now i'm gonna order those \\
\hline Lyrics $\rightarrow$ Joyce & And all the guns is in Kesha's name & All your gunsees belong to Kesha. \\
\hline $\mathrm{AAE} \rightarrow 1810$ & boredom gonna be the death of me one day & $\begin{array}{l}\text { I shall die, one day, of the insupportable want of amuse- } \\
\text { ment. }\end{array}$ \\
\hline $\mathrm{AAE} \rightarrow 1890$ & $\begin{array}{l}\text { That's just what I needed to see.... Thank Ya } \\
\text { Lord }\end{array}$ & Thank you, Lord; that is just what I was expecting. \\
\hline $\mathrm{AAE} \rightarrow$ Swit. & okay ii will see you later & yeah see you lat \\
\hline Poetry $\rightarrow$ Tweet & Fam'd heroes! had their royal home: & royal bloods heroes: \\
\hline Tweet $\rightarrow$ Bible & Check out this new painting that I uploaded to! & Look upon my new picture that I have set before thee! \\
\hline Swit. $\rightarrow$ Shak. & wear to work & And what dost thou wear for thy work? \\
\hline Tweet $\rightarrow$ Poetry & Now I gotta delete it & O now, must I part? And can I now erase \\
\hline Tweet $\rightarrow 1810$. & $\begin{array}{l}\text { \#India is now producing the worlds cheapest } \\
\text { solar power \#energy }\end{array}$ & Now is India's solar power cheapest of all the world. \\
\hline Poetry $\rightarrow$ Joyce & Away, away, or I shall dearly rue & O offside, away, or do I am rather sad. \\
\hline Tweet $\rightarrow$ Swit. & Oh shit ima be a senior & so uh i got to the senior level of the business \\
\hline
\end{tabular}

Table 9: More example outputs from our model STRAP trained on our dataset CDS. Our project page will provide all 110k style transferred outputs generated by STRAP on CDS. 


\begin{tabular}{|c|c|c|c|c|c|c|c|c|c|c|}
\hline \multirow[t]{2}{*}{ Paper } & \multicolumn{5}{|c|}{ Automatic } & \multicolumn{5}{|c|}{ Human } \\
\hline & $\mathrm{ACC}$ & SIM & FL & $\mathrm{CA}$ & SA & $\mathrm{ACC}$ & SIM & FL & $\mathrm{CA}$ & SA \\
\hline Hu et al. (2017) & $\checkmark$ & & & & & & & & & \\
\hline Shen et al. (2017) & $\checkmark$ & & & & & $\checkmark$ & & $\checkmark$ & & $\mathrm{A} / \mathrm{B}$ \\
\hline Shetty et al. (2018) & $\checkmark$ & & & & & & $\mathrm{A} / \mathrm{B}$ & & & \\
\hline Fu et al. (2018) & $\checkmark$ & $\checkmark$ & & & & & $\checkmark$ & & & \\
\hline Li et al. (2018) & $\checkmark$ & $\checkmark$ & & & & $\checkmark$ & $\checkmark$ & $\checkmark$ & & $\checkmark$ \\
\hline Zhang et al. (2018) & $\checkmark$ & $\checkmark$ & & & & $\checkmark$ & $\checkmark$ & $\checkmark$ & & $\checkmark$ \\
\hline Nogueira dos Santos et al. (2018) & $\checkmark$ & $\checkmark$ & $\checkmark$ & & & & & & & \\
\hline Prabhumoye et al. (2018) & $\checkmark$ & & & & & & $\mathrm{A} / \mathrm{B}$ & $\checkmark$ & & \\
\hline Xu et al. (2018) & $\checkmark$ & $\checkmark$ & & $\checkmark$ & & $\checkmark$ & $\checkmark$ & & $\checkmark$ & \\
\hline Logeswaran et al. (2018) & $\checkmark$ & $\checkmark$ & $\checkmark$ & & & $\checkmark$ & $\checkmark$ & $\checkmark$ & & \\
\hline Yang et al. (2018) & $\checkmark$ & $\checkmark$ & $\checkmark$ & & & & & & & \\
\hline Subramanian et al. (2019) & $\checkmark$ & $\checkmark$ & $\checkmark$ & & & $\checkmark$ & $\checkmark$ & $\checkmark$ & & $\mathrm{A} / \mathrm{B}$ \\
\hline Luo et al. (2019) & $\checkmark$ & $\checkmark$ & & $\checkmark$ & & $\checkmark$ & $\checkmark$ & $\checkmark$ & $\checkmark$ & $\checkmark$ \\
\hline Pang and Gimpel (2019) & $\checkmark$ & $\checkmark$ & $\checkmark$ & $\checkmark$ & & $\mathrm{A} / \mathrm{B}$ & $\mathrm{A} / \mathrm{B}$ & $\mathrm{A} / \mathrm{B}$ & & $\mathrm{A} / \mathrm{B}$ \\
\hline Ma et al. (2019) & $\checkmark$ & $\checkmark$ & $\checkmark$ & & & $\checkmark$ & $\checkmark$ & $\checkmark$ & & \\
\hline Dai et al. (2019) & $\checkmark$ & $\checkmark$ & $\checkmark$ & & & $\mathrm{A} / \mathrm{B}$ & $\mathrm{A} / \mathrm{B}$ & $\mathrm{A} / \mathrm{B}$ & & \\
\hline Sudhakar et al. (2019) & $\checkmark$ & $\checkmark$ & $\checkmark$ & & & $\mathrm{A} / \mathrm{B}$ & $\mathrm{A} / \mathrm{B}$ & $\mathrm{A} / \mathrm{B}$ & & $\mathrm{A} / \mathrm{B}$ \\
\hline Mir et al. (2019) & $\checkmark$ & $\checkmark$ & $\checkmark$ & & & $\checkmark$ & $\checkmark$ & $\checkmark$ & & \\
\hline Gröndahl and Asokan (2019) & $\checkmark$ & $\checkmark$ & & & & & $\checkmark$ & & & \\
\hline Tikhonov et al. (2019) & $\checkmark$ & $\checkmark$ & & & & & & & & \\
\hline Syed et al. (2020) & $\checkmark$ & $\checkmark$ & & & & & & & & \\
\hline Madaan et al. (2020) & $\checkmark$ & $\checkmark$ & & & & $\checkmark$ & $\checkmark$ & $\checkmark$ & & \\
\hline He et al. (2020) & $\checkmark$ & $\checkmark$ & $\checkmark$ & & & & & & & \\
\hline Ours & $\checkmark$ & $\checkmark$ & $\checkmark$ & $\checkmark$ & $\checkmark$ & & $\checkmark$ & $\checkmark$ & & $\checkmark$ \\
\hline
\end{tabular}

Table 10: Survey of evaluation methods used in 23 prior papers. We check whether prior work evaluate their algorithm on transfer accuracy (ACC), semantic similarity (SIM), fluency (FL), corpus-level aggregation (CA) and sentence-level aggregation (SA). We use the "A/B" to denote relative comparisons via $\mathrm{A} / \mathrm{B}$ testing between generations from the baseline and the proposed system, rather than absolute performance numbers. Specific implementations of the metrics have been provided in Table 11. We do not include Pang (2019) since it's a survey of existing evaluation methods. 


\begin{tabular}{|c|c|c|c|c|c|c|}
\hline \multirow[t]{2}{*}{ Paper } & \multicolumn{3}{|c|}{ Automatic } & \multicolumn{3}{|c|}{ Human } \\
\hline & $\mathrm{ACC}$ & SIM & FL & $\mathrm{ACC}$ & SIM & FL \\
\hline Hu et al. (2017) & L-CNN & & & & & \\
\hline Shen et al. (2017) & $\mathrm{CNN}$ & & & Likert-4 & & Likert-4 \\
\hline Shetty et al. (2018) & RNN/CNN & METEOR & & & $\mathrm{A} / \mathrm{B}$ & \\
\hline Fu et al. (2018) & LSTM & GloVE & & & Likert-3 & \\
\hline Li et al. (2018) & LSTM & BLEU & & Likert-5 & Likert-5 & Likert-5 \\
\hline Zhang et al. (2018) & GRU & BLEU & & Likert-5 & Likert-5 & Likert-5 \\
\hline Nogueira dos Santos et al. (2018) & SVM & GloVE & PPL & & & \\
\hline Prabhumoye et al. (2018) & $\mathrm{CNN}$ & & & & $\mathrm{A} / \mathrm{B}$ & Likert-4 \\
\hline Xu et al. (2018) & $\mathrm{CNN}$ & BLEU & & Likert-10 & Likert-10 & \\
\hline Logeswaran et al. (2018) & $\mathrm{CNN}$ & BLEU & PPL & Likert-5 & Likert-5 & Likert-5 \\
\hline Yang et al. (2018) & $\mathrm{CNN}$ & BLEU & PPL & & & \\
\hline Subramanian et al. (2019) & fastText & BLEU & PPL & Binary & Likert-5 & Likert-5 \\
\hline Luo et al. (2019) & $\mathrm{CNN}$ & BLEU & & Likert-5 & Likert-5 & Likert-5 \\
\hline Pang and Gimpel (2019) & $\mathrm{CNN}$ & GloVE & PPL & $\mathrm{A} / \mathrm{B}$ & $\mathrm{A} / \mathrm{B}$ & $\mathrm{A} / \mathrm{B}$ \\
\hline Ma et al. (2019) & $\mathrm{CNN}$ & BLEU & PPL & Likert-5 & Likert-5 & Likert-5 \\
\hline Dai et al. (2019) & fastText & BLEU & PPL & $\mathrm{A} / \mathrm{B}$ & $\mathrm{A} / \mathrm{B}$ & $\mathrm{A} / \mathrm{B}$ \\
\hline Sudhakar et al. (2019) & fastText & GLEU & PPL & $\mathrm{A} / \mathrm{B}$ & $\mathrm{A} / \mathrm{B}$ & $\mathrm{A} / \mathrm{B}$ \\
\hline Mir et al. (2019) & EMD & GloVE* & Classify & Likert-5* & Likert-5* & Binary* \\
\hline Gröndahl and Asokan (2019) & LSTM/CNN & METEOR & & & & \\
\hline Tikhonov et al. (2019) & $\mathrm{CNN}$ & BLEU & & & & \\
\hline Syed et al. (2020) & FineGrain & BLEU & & & & \\
\hline Madaan et al. (2020) & AWD-LSTM & METEOR & & Likert-5 & Likert-5 & Likert-5 \\
\hline He et al. (2020) & $\mathrm{CNN}$ & BLEU & PPL & & & \\
\hline Ours & RoBERTa-L & SIM-PP & Classify & & Binary & Binary \\
\hline
\end{tabular}

Table 11: Survey of implementations of evaluation metrics to measure Accuracy (ACC), Similarity (SIM) and Fluency (FL) used in 23 prior papers. For a cleaner version of this table with aggregation information, see Table 10. The * marks in Mir et al. (2019) denote a carefully designed unique implementation. We do not include Pang (2019) since it's a survey of existing evaluation methods.

\begin{tabular}{lrrrrr|rrrrr}
\hline Model & \multicolumn{4}{c|}{ Formality } & \multicolumn{5}{c}{ Shakespeare } \\
& ACC & SIM & FL & GM(A,S,F) & $J(\mathrm{~A}, \mathrm{~S}, \mathrm{~F})$ & ACC & SIM & FL & GM(A,S,F) & $J(\mathrm{~A}, \mathrm{~S}, \mathrm{~F})$ \\
\hline COPY & 5.2 & 41.8 & 88.4 & 26.8 & 0.2 & 9.6 & 20.1 & 79.1 & 24.8 & 0.1 \\
NAÏVE & 49.7 & 22.1 & 89.4 & 44.4 & 2.4 & 49.9 & 10.5 & 78.9 & 34.6 & 1.1 \\
REF & 93.3 & 100 & 89.7 & 94.2 & 88.2 & 90.4 & 100 & 79.1 & 89.4 & 67.2 \\
\hline UNMT & 78.5 & 15.1 & 52.5 & 39.7 & 11.7 & 70.5 & 7.9 & 49.6 & 30.2 & 1.7 \\
DLSM & 78.0 & 18.5 & 53.7 & 42.6 & 9.5 & 71.1 & 12.5 & 49.4 & 35.2 & 2.0 \\
\hline STRAP $(p=0.0)$ & 67.7 & 28.8 & 90.4 & 56.1 & 19.3 & 71.7 & 10.3 & 85.2 & 39.8 & 5.9 \\
STRAP $(p=0.6)$ & 70.7 & 25.3 & 88.5 & 54.1 & 17.2 & 75.7 & 8.8 & 82.7 & 38.1 & 5.4 \\
STRAP $(p=0.9)$ & 76.8 & 17.0 & 77.4 & 46.6 & 12.2 & 79.8 & 6.1 & 71.7 & 32.7 & 3.4 \\
\hline
\end{tabular}

Table 12: A table equivalent to Table 1 but using BLEU scores for SIM instead of the paraphrase similarity model from Wieting et al. (2019). The Formality dataset had 4 available reference sentences whereas the Shakespeare dataset had only 1 available reference sentence. Our system STRAP significantly beats prior work (UNMT, DLSM) on $J(\cdot)$ metrics even with BLEU scores. 


\begin{tabular}{|c|c|c|c|c|c|c|c|c|}
\hline \multirow[t]{2}{*}{ Model } & \multirow[t]{2}{*}{$\operatorname{ACC}(\mathrm{A})$} & \multicolumn{2}{|c|}{$\operatorname{SIM}(S)$} & \multirow[t]{2}{*}{ FL (F) } & \multicolumn{2}{|c|}{$J(\mathrm{~A}, \mathrm{~S})$} & \multicolumn{2}{|c|}{$J(\mathrm{~A}, \mathrm{~S}, \mathrm{~F})$} \\
\hline & & BL & PP & & BL & PP & BL & PP \\
\hline COPY & 8.0 & 32.6 & 80.9 & 90.1 & 0.4 & 7.1 & 0.3 & 6.4 \\
\hline REF & 87.8 & 100 & 100 & 90.1 & 91.1 & 87.8 & 83.5 & 78.9 \\
\hline NAÏVE & 67.9 & 10.7 & 32.0 & 91.5 & 1.7 & 9.3 & 1.5 & 8.5 \\
\hline BT (Prabhumoye et al., 2018) & 47.4 & 1.3 & 21.1 & 8.0 & 0.7 & 11.4 & 0.0 & 1.3 \\
\hline MultiDec (Fu et al., 2018) & 26.0 & 12.0 & 36.9 & 15.1 & 1.4 & 8.9 & 0.0 & 1.5 \\
\hline Del. (Li et al., 2018) & 24.2 & 30.1 & 53.5 & 20.8 & 3.1 & 10.2 & 0.0 & 1.6 \\
\hline Unpaired (Xu et al., 2018) & 53.9 & 1.6 & 16.3 & 34.9 & 0.4 & 10.9 & 0.0 & 2.2 \\
\hline DelRetri. (Li et al., 2018) & 52.8 & 21.9 & 47.6 & 16.3 & 11.9 & 23.4 & 0.2 & 4.2 \\
\hline CrossAlign. (Shen et al., 2017) & 59.0 & 3.3 & 25.0 & 31.7 & 2.0 & 14.9 & 0.3 & 5.2 \\
\hline Retri. (Li et al., 2018) & 90.0 & 0.5 & 9.0 & 62.1 & 0.5 & 8.3 & 0.3 & 5.5 \\
\hline Templ. (Li et al., 2018) & 37.1 & 36.4 & 67.8 & 32.3 & 11.9 & 23.7 & 1.3 & 7.8 \\
\hline DualRL (Luo et al., 2019) & 51.8 & 45.0 & 65.1 & 59.0 & 14.6 & 29.9 & 8.1 & 21.7 \\
\hline UNMT (Zhang et al., 2018) & 64.5 & 34.4 & 64.8 & 45.9 & 28.2 & 41.2 & 14.7 & 22.1 \\
\hline STRAP $(p=0.0) *$ & 57.7 & 31.1 & 69.7 & 93.8 & 19.5 & 40.8 & 18.3 & 38.7 \\
\hline STRAP $(p=0.6)^{*}$ & 63.4 & 26.5 & 66.7 & 91.4 & 18.3 & 43.0 & 17.1 & $\mathbf{4 0 . 0}$ \\
\hline STRAP $(p=0.9)^{*}$ & 70.3 & 17.3 & 59.0 & 81.4 & 13.6 & 41.6 & 11.8 & 34.3 \\
\hline
\end{tabular}

Table 13: More comparisons against prior work on the Formality dataset (Rao and Tetreault, 2018) using the outputs provided in the publicly available codebase of Luo et al. (2019) using both BLEU score (BL) and paraphrase similarity (PP). This model uses the Family \& Relationships split of the Formality dataset whereas (He et al., 2020) used the Entertainment \& Music split. Hence, we have retrained our RoBERTa-large classifiers to reflect the new distribution. *Note: While our system significantly outperforms prior work, we re-use the formality system used in Table 1 and Table 2 for these results, which was trained on Entertainment \& Music (consistent with He et al. (2020)). There could be a training dataset mismatch between our model and the models from Luo et al. (2019), since the Formality dataset has two domains. This is not clarified in Luo et al. (2019) to the best of our knowledge. 


\begin{tabular}{|c|c|c|c|c|c|}
\hline Style & Train & Dev & Test & Source & Examples \\
\hline Shakespeare & 24,852 & 1,313 & 1,293 & $\begin{array}{l}\text { Shakespeare split of } \mathrm{Xu} \text { et al. } \\
\text { (2012). }\end{array}$ & $\begin{array}{l}\text { 1. Why, Romeo, art thou mad? } \\
\text { 2. I beseech you, follow straight. }\end{array}$ \\
\hline English Tweets & $5,164,874$ & 39,662 & 39,690 & $\begin{array}{l}\text { A random sample of English } \\
\text { tweets collected on 8th-9th July, } \\
2019 \text { using Twitter APIs. }\end{array}$ & $\begin{array}{l}\text { 1. Lol figures why I dont wanna } \\
\text { talk to anyone rn } \\
\text { 2. omg no problem i felt bad } \\
\text { holding it! i love youuuu }\end{array}$ \\
\hline Bible & 31,404 & 1,714 & 1,714 & $\begin{array}{l}\text { The English Bible collected } \\
\text { from Project Gutenberg (Hart, } \\
\text { 1992) (link). }\end{array}$ & $\begin{array}{l}\text { 1. Jesus saith unto her, Woman, } \\
\text { what have I to do with thee? } \\
\text { 2. Wherefore it is lawful to do } \\
\text { well on the sabbath days. }\end{array}$ \\
\hline $\begin{array}{l}\text { Romantic } \\
\text { Poetry }\end{array}$ & 26,880 & 1,464 & 1,470 & $\begin{array}{l}\text { The Romantic section of the Po- } \\
\text { etry bookshelf on Project Guten- } \\
\text { berg (link). }\end{array}$ & $\begin{array}{l}\text { 1. There in that forest did his } \\
\text { great love cease; } \\
\text { 2. But, oh! for Hogarth's magic } \\
\text { pow'r! }\end{array}$ \\
\hline Switchboard & 145,823 & 1,487 & 1,488 & $\begin{array}{l}\text { Conversational speech tran- } \\
\text { scripts (link) from the Switch- } \\
\text { board speech recognition } \\
\text { corpus (Godfrey et al., 1992). }\end{array}$ & $\begin{array}{l}\text { 1. uh-huh well we're not all like } \\
\text { that um } \\
\text { 2. well yes } i \text { i well } i \text { - } i \text { don't think } \\
i \text { have the time to really become } \\
\text { a student in every article }\end{array}$ \\
\hline $\begin{array}{l}\text { AAE (African } \\
\text { American } \\
\text { English) Tweets }\end{array}$ & 717,634 & 7,316 & 7,315 & $\begin{array}{l}\text { Using the geo-located tweet cor- } \\
\text { pus collected by Blodgett et al. } \\
(2016) \text {. }\end{array}$ & $\begin{array}{l}\text { 1. ay yall everything good we } \\
\text { did dat... } \\
\text { 2. I know data right, it don't get } \\
\text { more real than that. }\end{array}$ \\
\hline James Joyce & 37,082 & 2,054 & 2,043 & $\begin{array}{l}\text { Two novels (Ulysses, } \\
\text { Finnegans) of James Joyce from } \\
\text { Project Gutenberg (link) and the } \\
\text { Internet Archive (link). }\end{array}$ & $\begin{array}{l}\text { 1. At last she spotted a weeny } \\
\text { weeshy one miles away. } \\
\text { 2. chees of all chades at the } \\
\text { same time as he wags an an- } \\
\text { tomine art of being rude like the } \\
\text { boor. }\end{array}$ \\
\hline Lyrics & $4,588,522$ & 252,368 & 252,397 & $\begin{array}{l}\text { Music lyrics dataset from } \\
\text { MetroLyrics, used in a Kaggle } \\
\text { competition (link). }\end{array}$ & $\begin{array}{l}\text { 1. I gotta get my mind off you, } \\
\text { 2. This is it, we are, baby, we } \\
\text { are one of a kind }\end{array}$ \\
\hline $\begin{array}{l}1810-1830 \text { his- } \\
\text { torical English }\end{array}$ & 205,286 & 5,340 & 5,338 & $\begin{array}{l}1810-1830 \text { in the Corpus } \\
\text { of Historical American En- } \\
\text { glish (Davies, 2012) using } \\
\text { fiction, non-fiction and maga- } \\
\text { zine domains (link). }\end{array}$ & $\begin{array}{l}\text { 1. The fulness of my fancy ren- } \\
\text { ders my eye vacant and inactive. } \\
\text { 2. What then do you come hither } \\
\text { for at such an hour? }\end{array}$ \\
\hline $\begin{array}{l}\text { 1890-1910 his- } \\
\text { torical English }\end{array}$ & $1,210,687$ & 32,024 & 32,018 & $\begin{array}{l}1890-1910 \text { in the Corpus of His- } \\
\text { torical American English using } \\
\text { fiction, non-fiction and maga- } \\
\text { zine domains (link). }\end{array}$ & $\begin{array}{l}\text { 1. Nor shall I reveal the name } \\
\text { of my friend; I do not wish to } \\
\text { expose him to a torrent of abuse. } \\
\text { 2. You know olive oil don't give } \\
\text { the brightest illumination. }\end{array}$ \\
\hline $\begin{array}{l}\text { 1990-2010 his- } \\
\text { torical English }\end{array}$ & $1,865,687$ & 48,985 & 48,982 & $\begin{array}{l}1990-2010 \text { in the Corpus of His- } \\
\text { torical American English using } \\
\text { fiction, non-fiction and maga- } \\
\text { zine domains (link). }\end{array}$ & $\begin{array}{l}\text { 1. They were, in fact, tears of } \\
\text { genuine relief. } \\
\text { 2. I don't know why, but I sensed } \\
\text { there was something wrong. }\end{array}$ \\
\hline Total & $14,018,731$ & 393,727 & 393,748 & & \\
\hline
\end{tabular}

Table 14: Details of our new benchmark dataset CDS along with representative examples. Our dataset contains eleven lexically and syntactically diverse styles and has a total of nearly $15 \mathrm{M}$ sentences, an order of magnitude larger than previous datasets. We will provide more representative examples along with our entire dataset in the project page http: / / style.cs.umass.edu. 


\begin{tabular}{|c|c|c|c|c|c|c|c|c|}
\hline Split & Orig. ACC & Orig. FL & Model & $\operatorname{ACC}(\mathrm{A})$ & SIM (S) & FL (F) & $J(\mathrm{~A}, \mathrm{~S})$ & $J(\mathrm{~A}, \mathrm{~S}, \mathrm{~F})$ \\
\hline \multirow[t]{3}{*}{ AAE Tweets } & 87.6 & 56.4 & Ours $(p=0.0)$ & 21.0 & 70.1 & 71.6 & 12.6 & 8.3 \\
\hline & & & Ours $(p=0.6)$ & 32.5 & 65.7 & 63.5 & 18.3 & 10.2 \\
\hline & & & Ours $(p=0.9)$ & 46.1 & 57.8 & 45.9 & 23.6 & 9.8 \\
\hline \multirow[t]{3}{*}{ Bible } & 98.3 & 87.5 & Ours $(p=0.0)$ & 48.0 & 58.4 & 81.2 & 24.7 & 20.9 \\
\hline & & & Ours $(p=0.6)$ & 52.5 & 55.1 & 79.8 & 25.7 & 21.3 \\
\hline & & & Ours $(p=0.9)$ & 56.9 & 49.4 & 74.0 & 25.3 & 19.3 \\
\hline \multirow[t]{3}{*}{ COHA 1810s-1820s } & 83.0 & 89.1 & Ours $(p=0.0)$ & 25.9 & 66.5 & 84.5 & 16.4 & 13.7 \\
\hline & & & Ours $(p=0.6)$ & 34.0 & 63.0 & 81.5 & 20.1 & 16.0 \\
\hline & & & Ours $(p=0.9)$ & 42.7 & 57.3 & 73.6 & 22.9 & 16.5 \\
\hline \multirow[t]{3}{*}{ COHA 1890s-1900s } & 76.5 & 91.2 & Ours $(p=0.0)$ & 36.1 & 68.9 & 86.7 & 23.7 & 21.2 \\
\hline & & & Ours $(p=0.6)$ & 41.1 & 65.7 & 83.8 & 25.5 & 22.1 \\
\hline & & & Ours $(p=0.9)$ & 44.3 & 59.4 & 72.0 & 25.0 & 19.2 \\
\hline \multirow[t]{3}{*}{ COHA 1990s-2000s } & 86.9 & 96.8 & Ours $(p=0.0)$ & 40.4 & 69.0 & 87.7 & 26.6 & 24.4 \\
\hline & & & Ours $(p=0.6)$ & 46.1 & 65.6 & 86.0 & 28.9 & 26.3 \\
\hline & & & Ours $(p=0.9)$ & 46.1 & 59.4 & 76.1 & 26.1 & 21.7 \\
\hline \multirow[t]{3}{*}{ English Tweets } & 80.7 & 79.9 & Ours $(p=0.0)$ & 20.0 & 71.0 & 79.1 & 13.5 & 11.0 \\
\hline & & & Ours $(p=0.6)$ & 28.9 & 67.5 & 72.2 & 18.1 & 13.7 \\
\hline & & & Ours $(p=0.9)$ & 40.8 & 60.0 & 55.5 & 22.7 & 13.4 \\
\hline \multirow[t]{3}{*}{ James Joyce } & 87.1 & 48.2 & Ours $(p=0.0)$ & 43.0 & 69.6 & 79.8 & 28.7 & 22.0 \\
\hline & & & Ours $(p=0.6)$ & 52.2 & 63.7 & 62.8 & 32.0 & 29.6 \\
\hline & & & Ours $(p=0.9)$ & 63.6 & 54.8 & 40.5 & 33.5 & 11.3 \\
\hline \multirow[t]{3}{*}{ Lyrics } & 88.7 & 78.9 & Ours $(p=0.0)$ & 51.9 & 71.6 & 79.4 & 35.6 & 29.0 \\
\hline & & & Ours $(p=0.6)$ & 53.4 & 68.6 & 71.4 & 34.8 & 26.0 \\
\hline & & & Ours $(p=0.9)$ & 53.3 & 62.1 & 51.9 & 31.4 & 18.1 \\
\hline \multirow[t]{3}{*}{ Romantic Poetry } & 93.8 & 40.2 & Ours $(p=0.0)$ & 55.0 & 63.8 & 58.9 & 33.5 & 17.2 \\
\hline & & & Ours $(p=0.6)$ & 62.4 & 60.3 & 51.8 & 35.6 & 16.2 \\
\hline & & & Ours $(p=0.9)$ & 69.8 & 55.3 & 40.3 & 36.8 & 13.0 \\
\hline \multirow[t]{3}{*}{ Shakespeare } & 86.1 & 59.9 & Ours $(p=0.0)$ & 36.8 & 65.5 & 76.9 & 21.7 & 15.4 \\
\hline & & & Ours $(p=0.6)$ & 52.1 & 58.6 & 65.4 & 28.2 & 16.6 \\
\hline & & & Ours $(p=0.9)$ & 63.7 & 48.9 & 44.2 & 29.3 & 11.3 \\
\hline \multirow[t]{3}{*}{ Switchboard } & 99.7 & 63.1 & Ours $(p=0.0)$ & 62.9 & 67.4 & 77.0 & 40.8 & 32.0 \\
\hline & & & Ours $(p=0.6)$ & 77.2 & 63.7 & 64.2 & 47.5 & 30.2 \\
\hline & & & Ours $(p=0.9)$ & 84.9 & 56.6 & 44.0 & 46.8 & 20.1 \\
\hline \multirow[t]{3}{*}{ Overall } & 88.0 & 71.9 & Ours $(p=0.0)$ & 40.1 & 67.4 & 78.4 & 25.3 & 19.6 \\
\hline & & & Ours $(p=0.6)$ & 48.4 & 63.4 & 71.1 & 28.6 & 20.7 \\
\hline & & & Ours $(p=0.9)$ & 55.7 & 56.5 & 56.2 & 29.4 & 15.8 \\
\hline
\end{tabular}

Table 15: A detailed performance breakup when transferring to each style in CDS from the other 10 styles. We test three nucleus sampling (Holtzman et al., 2020) strategies with our trained model by varying the $p$ value between 0.0 (greedy) and 1.0 (full sampling). For reference, the classification accuracy (Orig. ACC) and fluency (Orig. FL) of original sentences in the target style corpus are provided. 


\begin{tabular}{lrrr|rrr}
\hline \multirow{2}{*}{ Split } & \multicolumn{3}{c|}{ Diverse Paraphraser } & \multicolumn{3}{c}{ Non-Diverse Paraphraser } \\
& Similarity $(\uparrow)$ & Lexical $(\downarrow)$ & Syntactic $(\downarrow)$ & Similarity $(\uparrow)$ & Lexical $(\downarrow)$ & Syntactic $(\downarrow)$ \\
\hline AAE Tweets & 65.1 & 44.7 & 0.43 & 74.3 & 66.4 & 0.82 \\
Bible & 74.6 & 48.5 & 0.55 & 88.3 & 73.5 & 0.92 \\
COHA 1810s-1820s & 74.0 & 50.6 & 0.51 & 86.3 & 71.8 & 0.92 \\
COHA 1890s-1900s & 75.3 & 52.0 & 0.50 & 88.2 & 75.3 & 0.93 \\
COHA 1990s-2000s & 77.6 & 57.4 & 0.53 & 89.9 & 80.7 & 0.95 \\
English Tweets & 73.1 & 52.4 & 0.50 & 82.8 & 75.7 & 0.91 \\
James Joyce & 71.5 & 47.8 & 0.35 & 82.4 & 69.8 & 0.82 \\
Lyrics & 74.5 & 52.8 & 0.52 & 86.7 & 78.6 & 0.92 \\
Romantic Poetry & 72.3 & 46.3 & 0.44 & 81.3 & 67.1 & 0.86 \\
Shakespeare & 67.9 & 38.7 & 0.23 & 81.4 & 63.4 & 0.75 \\
Switchboard & 71.6 & 50.1 & 0.55 & 81.1 & 72.4 & 0.90 \\
\hline Overall & 72.5 & 49.2 & 0.46 & 83.9 & 72.3 & 0.88 \\
\hline
\end{tabular}

Table 16: A detailed style-wise breakup of the diverse paraphrase quality in CDS (the training data for the inverse paraphrase model, described in Section 2.1 and Section 2.4). The ideal paraphraser should score lower on "Lexical" and "Syntactic" overlap and high on "Similiarity". Overall, our method achieves high diversity (51\% unigram change and $27 \%$ word shuffling, compared to $28 \%$ unigram and $6 \%$ shuffling for non-diverse), while maintaining good semantic similarity ( $\mathrm{SIM}=72.5$ vs 83.9 for non-diverse) even in complex stylistic settings. We measure lexical overlap in terms of unigram F1 overlap using the evaluation scripts from Rajpurkar et al. (2016). Syntactic overlap is measured using Kendall's $\tau_{\mathrm{B}}$ (Kendall, 1938) of shared vocabulary. A $\tau_{\mathrm{B}}=1.0$ indicates no shuffling whereas a value of $\tau_{\mathrm{B}}=-1.0$ indicates $100 \%$ shuffling (complete reversal). Finally, the SIM model from Wieting et al. (2019) is used for measuring similarity. 